\title{
Interleukin-6 mediates PSAT1 expression and serine metabolism in TSC2-deficient cells
}

Ji Wang ${ }^{1 *}$, Harilaos Filippakis ${ }^{1 *}$, Thomas Hougard ${ }^{1}$, Heng Du$^{1}$, Chenyang Ye ${ }^{2}$, Heng-Jia Liu ${ }^{1}$, Long Zhang ${ }^{1}$, Khadijah Hindi' ${ }^{1}$, Shefali Bagwe ${ }^{1}$, Julie Nijmeh ${ }^{1}$, John M. Asara ${ }^{3}$, Wei Shi ${ }^{4}$, Souheil El-Chemaly ${ }^{1}$, Elizabeth P. Henske ${ }^{1 \#}$, Hilaire C. Lam ${ }^{1 \#}$

${ }^{1}$ Pulmonary and Critical Care Medicine, Brigham and Women's Hospital, Harvard Medical School, Boston, MA 02115

${ }^{2}$ The F.M. Kirby Neurobiology Center, Boston Children's Hospital, Department of Neurology, Harvard Medical School, Boston, MA 02115

${ }^{3}$ Division of Signal Transduction, Beth Israel Deaconess Medical Center and Department of Medicine, Harvard Medical School Boston, MA, USA 02215

${ }^{4}$ Department of Surgery, Children's Hospital Los Angeles, Keck School of Medicine, University of Southern California, Los Angeles, CA 90027

${ }^{*}$ Authors contributed equally to this work

\# Co-corresponding authors:

Hilaire C. Lam

Email: hclam@bwh.harvard.edu

Elizabeth P. Henske

Email: ehenske@bwh.harvard.edu

Author Contributions: E.P. Henske and H.C. Lam conceived and designed the study. J. Wang, H. Filippakis, T. Hougard, H. Du, C. Ye, H. Liu, L. Zhang, K. Hindi, performed experiments and assisted in data analysis. S. Bagwe and S. El-Chemaly assisted with human samples and data interpretation. J.M. Asara and W. Shi assisted with expert technical advice and data interpretation. J. Wang, H. Filippakis, J. Nijmeh, E.P. Henske, and H.C. Lam wrote and edited the manuscript.

Competing Interest Statement: The authors declare no potential conflicts of interest.

Classification: Major category: Biological Sciences Minor category: Cell Biology

Keywords: Tuberous sclerosis complex (TSC) / Lymphangioleiomyomatosis (LAM), mTORC1/ Rapamycin, Interleukin-6 (IL-6), serine metabolism, phosphoserine aminotransferase 1 (PSAT1) 


\begin{abstract}
Tuberous sclerosis complex (TSC) and lymphangioleiomyomatosis (LAM) are caused by aberrant mechanistic Target of Rapamycin Complex 1 (mTORC1) activation due to loss of either TSC1 or TSC2. Cytokine profiling of TSC2-deficient LAM patient-derived cells revealed striking upregulation of Interleukin-6 (IL-6). LAM patient plasma contained increased circulating IL-6 compared with healthy controls, and TSC2-deficient cells showed upregulation of IL-6 transcription and secretion compared to wildtype cells. IL-6 blockade repressed the proliferation and migration of TSC2-deficient cells and reduced oxygen consumption and extracellular acidification. U-13 C glucose tracing revealed that IL-6 knockout reduced 3-phosphoserine and serine production in TSC2-deficient cells, implicating IL-6 in de novo serine metabolism. IL-6 knockout reduced expression of phosphoserine aminotransferase 1 (PSAT1), an essential enzyme in serine biosynthesis. Importantly, recombinant IL-6 treatment rescued PSAT1 expression in the TSC2-deficient, IL-6 knockout clones selectively and had no effect on wildtype cells. Treatment with anti-IL-6 (alL-6) antibody similarly reduced cell proliferation and migration and reduced renal tumors in $T s c 2^{+/-}$mice, while reducing PSAT1 expression. These data reveal a novel mechanism through which IL-6 regulates serine biosynthesis, with potential relevance to the therapy of tumors with mTORC1 hyperactivity.
\end{abstract}




\section{Introduction}

Tuberous Sclerosis Complex (TSC) is an autosomal dominant tumor suppressor syndrome that affects one in 10,000 infants (1-4). The majority of patients suffer from neurodevelopmental conditions including epilepsy, autism and cognitive impairment. Neoplastic lesions in the brain, skin, heart, kidney and lung are the primary causes of patient morbidity and mortality, particularly later in life $(5,6)$. Renal angiomyolipomas affect $\sim 70 \%$ of patients by 10 years of age (7). Lymphangioleiomyomatosis (LAM), characterized by pulmonary nodules and irreversible progressive cystic lung destruction, almost exclusively affects females with TSC and can also affect women with sporadic $\operatorname{LAM}(4,8)$.

TSC is caused by inactivating mutations in TSC1 or TSC2, resulting in aberrant activation of mechanistic Target of Rapamycin Complex 1 (mTORC1), a master regulator of cellular metabolism (9). Constitutive mTORC1 activation leads to extensive changes in signaling pathways and promotes lipid, nucleotide and protein biosynthesis contributing to the dysregulated growth and proliferation of cells in patients with TSC $(10,11)$. mTORC1 can be directly targeted by the allosteric inhibitor rapamycin and related analogs, "rapalogs," as well as catalytic mTOR inhibitors. Since mTORC1 inhibition primarily exerts cytostatic effects $(7,12)$, identifying novel therapeutic targets that can yield more durable or cytotoxic clinical responses is a key focus of ongoing TSC research.

Interleukin-6 (IL-6) is a secreted cytokine and critical mediator of inflammation (13). IL-6 binds to either soluble or membrane bound IL-6 receptor $\alpha$. The IL-6/IL-6R $\alpha$ complex then interacts with gp130 to activate signaling via Janus Kinase / Signal Transducer and Activator of Transcription 3 (JAK/STAT3). STAT3 regulates the transcription of hundreds of genes, including IL-6. This positive feedback loop has been previously shown to be an epigenetic mechanism of transformation downstream of transient Ras activation (14). STAT3 activation by mTORC1 is a well described feature of TSC lesions, along with an increase in IL-6 production (15-23). 
In support of prior findings, we find that IL-6 is upregulated in the plasma of patients with LAM and in preclinical models of TSC, in a TSC2- and mTORC1-dependent manner. By inhibiting IL-6 both genetically and with neutralizing antibodies, we find that TSC2-deficient cells depend on IL-6 to support the cell-autonomous metabolic reprogramming necessary for proliferation and migration. In particular, we implicate IL-6 in the regulation of de novo serine synthesis in TSC2deficient cells. The enzymatic reactions necessary for de novo serine metabolism produce serine, as well as antioxidants and the tricarboxylic acid (TCA) cycle intermediate, a-ketoglutarate. Specifically, the first rate-limiting step is executed by phosphoglycerate dehydrogenase (PHGDH), which converts the glycolytic intermediate 3-phoshphogylcerate to 3phosphohydroxypyruvate, regenerating NADH from NAD. The next step is mediated by phosphoserine aminotransferase 1 (PSAT1), which converts 3-phosphoydroxypyruvate to 3phosphoserine by transferring the amino group from glutamate and producing the TCA cycle intermediate a-ketoglutarate. Finally, 3-phosphoserine is converted to serine by phosphoserine phosphatase (PSPH). Serine can also be generated from glycine via the reversible action of serine hydroxymethyltransferase (SHMT) enzymes. Recent reviews have highlighted the importance of de novo serine metabolism in cancer survival and progression $(24,25)$. Our data implicate IL-6 as a novel regulator of de novo serine metabolism in mTORC1 hyperactive cells, thereby promoting the tumorigenic potential of TSC2-deficient cells.

\section{Results}

\section{IL-6 is upregulated in LAM patient plasma and preclinical LAM and TSC models}

Previous studies have shown that TSC2-deficient cells have a unique secretome, which may support the proliferation and metastatic potential of TSC tumors and LAM nodules by both cell autonomous and paracrine effects $(21-23,26)$. We performed a cytokine array using the TSC2-deficient 621-101 cell line, derived from a human angiomyolipoma, in comparison to the human embryonic kidney cell line, HEK293. The most robustly upregulated cytokine in 621-101 cells was IL-6 (Fig. S1A-D), a factor previously reported in LAM in vivo models and patient- 
derived cells $(21,23)$. We discovered that IL-6 is also upregulated in plasma from LAM patients compared to healthy controls (Fig. 1A).

We next confirmed that IL-6 expression is TSC2-dependent by comparing empty vector (621-102) or TSC2-addback cells (621-103) derived from the parental angiomyolipoma 621-101 cell line. TSC2 re-expression significantly reduced IL-6 mRNA expression and secretion of IL-6 $(\sim 70 \%, p<0.0001)$ compared to the TSC2-deficient lines (Fig. 1B and C). We next determined the expression and secretion of IL-6 in two additional pairs of TSC2-deficient and expressing cell lines: TTJ cells, derived from a renal tumor of a $T s c 2^{+/-}$mouse, expressing either empty vector or re-expressing TSC2, and $T s c 2^{+/+}$and $T s c 2^{-/-}$mouse embryonic fibroblasts (MEFs) $(27,28)$. IL-6 mRNA expression was upregulated by 7-fold in the TSC2-deficient TTJ cells relative to TSC2expressing control cells ( $p<0.01$, Fig. S1E) and by $\sim 30$-fold in the TSC2-deficient MEFs relative to TSC2-expressign MEFs ( $p<0.001$, Fig. 1D). Secretion of IL-6 was also increased in the TTJ cells ( $p<0.05$, Fig. S1F) and TSC2-deficient MEFs ( $p<0.0001$, Fig. 1E). Rapamycin (20nM, 24h) partially reduced IL-6 secretion by $\sim 50 \%(\mathrm{p}<0.001)$ in TSC2-deficient MEFs (Fig. 1E). Importantly, we found that IL-6 receptor $\alpha(\mathrm{IL}-6 \mathrm{R} \alpha)$ is upregulated in the membrane fraction of TSC2-deficient compared to TSC2-expressing MEFs, suggesting that IL-6 can act in an autocrine manner (Fig. 1F). IL-6 expression was also significantly elevated $\sim 2.5$-fold $(p<0.001)$ in kidney homogenates of CAGGCre-ER $R^{T M+-} ; T s C 2^{f f f}$ mice, which are characterized by cystic kidney disease driven mTORC1 hyperactivation, compared to CAGGCre-ERTM-/; Tsc2fff control mice (Fig.1G-I) (29, 30).

In summary, these data show that IL-6 is upregulated in patient plasma and consistently across numerous in vitro and in vivo models of TSC and LAM. Furthermore, IL-6 expression is both TSC2- and mTORC1-dependent. Finally, the increased expression of IL-6Ra on the TSC2deficient cells suggests that IL-6 may be secreted and detected by TSC2-deficient cells thereby exerting cell autonomous effects. 


\section{IL-6 knockout suppresses proliferation, migration, and induces a metabolic quiescent state in TSC2-deficient cells}

To investigate the dependance of TSC2-deficient cells on IL-6, we used CRISPR/Cas9 to knockout IL-6 from TSC2-deficient MEFs. We validated the knockout by measuring IL-6 secretion in three separate single cell clones generated from a single CRISPR/Cas9 guide (Fig. 2A). Genetic knockout of IL-6 decreased the proliferation of TSC2-deficient cells in serum-free conditions in comparison to IL-6 expressing TSC2-deficient cells $(>30 \%, p<0.001)$ as assessed by crystal violet staining as an indicator of cell density changes over 3 days (Fig. 2B). In order to maximize the differences between TSC2-deficient and wildtype control cells and to eliminate the possibility of the cells reacting to bovine IL-6 in the serum, we chose serum-free conditions for all subsequent experiments. Since metastasis is a key aspect of LAM pathogenesis, we also wanted to investigate the impact of IL-6 on the migration capacity of TSC2-deficient cells $(31,32)$. We discovered that IL-6 knockout also suppressed migration of TSC2-deficient cells through transwells towards a chemoattractant and in wound healing assays compared to control cells (Fig. 2C, D, and S2A, B). Importantly, conditioned media from TSC2-deficient cells with intact IL6 rescued proliferation of the IL-6 knockout cells (Fig. S2C). Treatment with rIL-6 (200 pg/ml) rescued the proliferation and migration of the TSC2 deficient cells with IL-6 knockout (Fig. S2D and $E$ )

To elucidate the mechanisms through which IL-6 knockout inhibits the proliferation of TSC2-deficient cells, we examined oxidative phosphorylation and glycolysis using the Seahorse XF Analyzer. The MitoStress Test Assay utilizes the effects of various mitochondrial targeted compounds on oxygen consumption rate $(\mathrm{OCR})$ and extracellular acidification rate (ECAR) as a readout of the cellular potential for oxidative phosphorylation (OXPHOS) and aerobic glycolysis, respectively. Prior studies have shown that TSC2-deficient cells upregulate glycolysis and OXPHOS to sustain the high bioenergetic and anabolic demands of mTORC1 hyperactivation (33-35). We found an overall decrease in OCR in the IL-6 knockout, TSC2-deficient cells 
compared to TSC2-deficient control cells expressing IL-6 (Fig. 2E). Statistical analysis of the OCR data demonstrated that both basal and maximal respiration (FCCP-induced) were significantly reduced $\sim 50 \%(p<0.001)$ in all three IL-6 knockout clones (Fig. $2 F)$. We also found that IL-6 knockout suppressed aerobic glycolysis by $\sim 50 \%$ ( $p<0.001$, Fig. $2 G)$. The reduction in both OCR and ECAR suggests that IL-6 knockout shifts TSC2-deficient cells to a bioenergetic quiescent state (Fig. $2 H$ ). Acute treatment with recombinant IL-6 (200pg/ml, 24 hours) had no impact on the OCR or glycolytic profile of IL-6 KO cells (Fig. S2F and G).

Collectively, these data demonstrate that IL-6 knockout has a significant impact on proliferation, migration, and metabolism of TSC2-deficient cells, suggesting that IL-6 may be a previously unappreciated mediator of metabolic reprogramming in TSC.

\section{IL-6 promotes de novo serine synthesis in TSC2-deficient cells}

Since we observed an IL-6 dependent reduction in oxidative phosphorylation and glycolysis, we investigated the role of IL-6 in TSC2-deficient cell metabolism by performing targeted metabolomics, measuring $\sim 270$ unique metabolites by liquid chromatography/mass spectrometry (LC/MS) (36). The IL-6 knockout clones showed a distinctive metabolic signature (Fig. S3A). There was some variability between the three clones, as expected from the process of single cell cloning; however, principal component analysis (PCA) confirmed that the clones were more similar to one another than the TSC2-deficient control cell line (Fig. S3B).

We next performed metabolite set enrichment analysis using MetaboAnalyst software by curating a single list of consistently differentially regulated metabolites from pairwise comparisons of the control cells with each of the IL-6 knockout clones. The most significantly impacted pathway $(p<0.0003)$ with a false discovery rate $(F D R)$ less than $5 \%$ was glycine and serine metabolism (Fig. S3C). Glycolytic intermediates can be diverted from the TCA Cycle for utilization in de novo serine synthesis and the Pentose Phosphate Pathway (PPP). In tumors, upregulation 
of de novo serine metabolism and PPP supports nucleotide metabolism and redox homeostasis $(37,38)$. TSC2-deficient cells with IL-6 knockout have a >2-fold reduction in 3-phosphoserine $(p<0.001$, Fig. S4A) and a $\sim 25 \%$ reduction in total serine levels $(p<0.0001$, Fig. S4B). Glycine was not measured in our samples and cysteine was not changed by knocking out IL-6 (Fig. S4C). We also discovered a $\sim 40 \%$ reduction in ribose 5-phosphate, a PPP intermediate, and $\sim 50 \%$ reduction in purines following IL-6 knockout in TSC2-deficient cells (Fig. S4D and E).

While early metabolites of the TCA cycle (citrate, aconitate, and isocitrate) were increased by $>2$-fold (Fig. S4F-H), metabolites downstream of $\alpha$-ketoglutarate, which can be produced by glutaminolysis, were unaffected or decreased by IL-6 loss (Fig. S4I-M). One possible explanation for this result is that decreased flux of glycolytic intermediates into PPP and de novo serine synthesis following IL-6 knockout increases flux into the TCA cycle. These data suggest that IL-6 plays significant role in the metabolism of TSC2-deficient cells, particularly glucose utilization.

To further investigate the impact of IL-6 on glucose metabolism, we used $\mathrm{U}-{ }^{13} \mathrm{C}$ glucose tracing and measured labeled metabolites after 0, 1, 3 and 24 hours (39), focusing on the fractional enrichment of metabolites directly related to glucose metabolism (Fig. 3A). All statistical analysis was performed at the final 24-hour time point shown in the bar graph below the time course data. The M+3 forms of serine (Fig. 3B) and 3-phosphoserine (Fig. 3C) were decreased in the cells with IL- 6 knockout compared to controls with intact IL- 6 by $\sim 50 \%$ $(p<0.0001)$. Enrichment of M+3 phosphoglycerate was equal between the IL-6 knockout lines and TSC2-deficient controls, suggesting that IL-6 selectively effects shuttling of glycolytic intermediates into the de novo serine synthesis pathways (Fig. 3D). IL-6 knockout decreased glucose-derived $M+2 \alpha$-ketoglutarate by $\sim 50 \%$ ( $p<0.001$, Fig. 3E) and increased glucose-derived $M+2$ glutamate by $\sim 10 \%(p<0.01$, Fig. $3 F)$.

Glucose tracing data provide insights into the steady-state targeted metabolomics data, highlighting a significant reduction in the TCA cycle intermediate a-ketoglutarate derived from 
glucose. Furthermore, knocking out IL-6 in TSC2-deficient cells selectively suppressed glucosederived intermediates from shuttling into de novo serine synthesis thereby impacting the production of serine.

\section{PSAT1 rescues proliferation of TSC2-deficient cells following IL-6 knockout}

To identify the potential molecular mechanisms through which IL-6 regulates de novo serine synthesis in TSC2-deficient cells, we measured the mRNA expression of the key metabolic enzymes of the pathway, PHGDH, PSAT1 and PSPH (Fig. 4A-D). All three of the enzymes were significantly reduced at the mRNA level $(>30 \%, p<0.01)$ in the IL- 6 knockout clones compared to the control TSC2-deficient cells.

We next confirmed the dependency of PSAT1 on IL-6 signaling in the TSC2-deficient cells using siRNA for IL-6Ra. A 90\% reduction in IL-6Ra significantly reduced PSAT1 expression $\sim 20 \%$ ( $p<0.01$, Fig. S5A). Interestingly, knocking down IL-6 $\sim 80 \%$ with siRNA had no effect on PSAT1 expression (Fig. S5B), suggesting that TSC2-deficient cells are responding at least in part to binding of extracellular IL-6 to the IL6-Ra in a cell autonomous manner to regulate PSAT1 and de novo serine biosynthesis.

Importantly, treatment with recombinant IL-6 (rIL-6) rescued the expression of PSAT1 in the knockout clones, inducing PSAT1 expression by $>3$-fold ( $p<0.0001$, Fig. 5E). Recombinant IL-6 had no effect on de novo serine synthesis enzymes in the TSC2-wildtype MEFs (Fig. S5C). Recombinant IL-6 also induced a >1.5-fold increase in PHGDH, PSPH and cytosolic SHMT1, but not mitochondrially localized SHMT2 (Fig. S5D and E). These data highlight the regulation of de novo serine synthesis by IL-6 selectively in TSC2-deficient cells. IL-6 knockout reduced PSAT1 protein expression $>25 \%$, while $\mathrm{PHGDH}$ and $\mathrm{PSPH}$ protein expression were unchanged compared to the control TSC2-deficient MEFs (Fig. 4F-I). 
In order to gain insights into the mechanisms by which IL-6 may regulate the enzymes of de novo serine synthesis, we performed additional knockdown experiments. Using STAT3 siRNA and inducible Raptor and Rictor knockout MEFs (40), we determined that PSAT1 is regulated in a STAT3-independent and mTORC1-dependent manner (Fig. S6A-C). These data are consistent with the finding that PSAT1 mRNA is increased $\sim 3$-fold in TSC2-deficient cells compared to wildtype control cells (Fig. S6D and E), as described in previous literature reports (41). In these prior publications, activating transcription factor 4 (ATF4), has been implicated in PSAT1 regulation downstream of mTORC1. We discovered a $\sim 15 \%$ decrease in ATF4 mRNA by IL-6 knockout $(p<0.001$, Fig. S6F). Interestingly, ATF4 protein expression and phosphorylation of S6 kinase were decreased in IL-6 knockout cells, compared to IL-6 expressing cells (Fig. S6G). Importantly, ATF4 expression was induced $\sim 2$-fold $(p<0.001)$ by rIL-6 treatment in IL-6 knockout TSC2-deficient MEFs compared to TSC2-wildtype MEFs, in which ATF4 expression was unchanged by rlL-6 (Fig. S6H and I). In order to determine the dependence of TSC2-deficient cells on PSAT1 downstream of IL-6, we overexpressed PSAT1 in the IL-6 knockout cells. Overexpression of PSAT1 was sufficient to rescue the proliferation of the IL-6 knockout cells and had no effect on the proliferation of the TSC2-deficient control cells (Fig. 4J and K).

In summary, these data suggest that IL-6 promotes the proliferation of TSC2-deficient cells in a cell autonomous manner via the upregulation of PSAT1 and induction of de novo serine synthesis.

\section{IL-6 neutralizing antibody suppresses PSAT1 expression, proliferation, and migration in TSC2-deficient cells}

IL-6 and IL-6Ra neutralizing antibodies are approved by the Food and Drug Administration of the United States for the treatment of various autoimmune diseases, including rheumatoid arthritis (42). Therefore, we sought to determine whether anti-IL-6 (alL-6) antibody treatment, would impact the proliferation and migration of TSC2-deficient cells. We used p- 
STAT3 ${ }^{\text {Y705 }}$ expression as a surrogate marker of alL- 6 antibody activity (Fig. S7A and B). In order to more closely mimic the long-term consequences of alL-6 antibody on the TSC2-deficient cells we treated cells for 14 days and then quantified colony formation in anchorage-independent soft agar conditions. alL-6 antibody reduced colony formation by $\sim 60 \%(p<0.01)$ compared to TSC2deficent cells treated with control IgG antibody (Fig. 5A and B). alL-6 antibody also acutely reduced the migration of TSC2-deficient cells $\sim 40 \%$ compared to IgG antibody control $(p<0.001$, Fig. $5 C$ and $D)$.

We next wanted to determine if alL-6 antibody treatment inhibited proliferation and migration effects by limiting de novo serine synthesis, as we observed in the IL-6 knockout cells. We discovered that alL-6 significantly reduced PSAT1 expression and reduced the $M+3$ labeling of serine in the TSC2-deficient cells (Fig. $5 E, F$ and S7C). These data suggest that targeting IL6 regulates PSAT1 expression and de novo serine synthesis in TSC2-deficient cells, and may be a therapeutic approach for the treatment of TSC and LAM.

\section{IL-6 neutralizing antibody suppresses the progression of renal tumors in $\mathrm{TSC2}^{+/-}$mice}

We next investigated the therapeutic impact of alL-6 antibody in $\mathrm{Tsc}^{+/-}$mice, a wellestablished preclinical model of TSC, which spontaneously develops renal cysts and cystadenomas by 6 months of age (43). In the first set of experiments, we determined the therapeutic benefits of alL-6 antibody as a single agent. Eight-month old $\mathrm{Tsc}^{+/-}$mice were treated with alL-6 antibody or control antibody (IgG) (200 ug, 3 times a week) for one month (Fig. $6 \mathrm{~A}$ ) and harvested $24-48 \mathrm{~h}$ after the final injection. Kidneys were inspected macroscopically for gross cysts and tumor burden (Fig. 6B). alL-6 antibody reduced both the gross and microscopic tumor burden $\sim 30 \%(p<0.05)$ compared to the IgG treated mice (Fig. $6 B-D)$. alL-6 antibody also reduced the number of proliferating Ki67+ cells in the renal lesions $(30 \%, p<0.01$, Fig. $6 E$ and $F)$. Semi-quantitative analysis of PSAT1 expression in renal lesions of the $\mathrm{Tsc}^{+/-}$mice was 
performed by a blinded observer. Two out of three alL-6 antibody treated mice showed a decrease in the PSAT1 expression across >10 lesions per mouse (Fig. S8A and B).

mTORC1 inhibition with rapamycin and related "rapalogs" is currently approved for patients with LAM and TSC. In LAM patients, lung function decline stabilizes, and many brain lesions and renal tumors shrink on mTORC1 inhibitor treatment in patients with TSC $(44,45)$. However, disease burden rapidly rebounds after treatment cessation, necessitating novel therapeutic interventions. To determine the lasting effectiveness of alL- 6 antibody treatment compared to rapamycin or combination therapy (Fig. 6G), Tsc2 ${ }^{+/-}$mice at 5-6 months of age were randomly assigned to receive lgG, alL-6 (200 ug, 3 times a week), rapamycin (3mg/kg 3 times a week) or the combination (alL-6 and rapamycin) for one month. The mice were harvested 2 months after the final treatment. All three treatment arms showed significant reduction in tumor burden compared to the IgG control mice (Fig. $6 \mathrm{H}$ and $I$ ). The mean gross tumor score was reduced by $25 \%$ by alL- 6 antibody $(p<0.01), 30 \%$ by rapamycin $(p<0.0001)$, and $50 \%$ by the combination $(p<0.0001)$ compared to the IgG treated controls. The mean microscopic tumor burden was reduced by $40 \%$ by alL- 6 antibody $(p<0.01), 60 \%$ by rapamycin $(p<0.0001)$, and $70 \%$ by the combination $(p<0.0001)$ compared to the $\lg G$ treated controls.

In summary, we observed a significant benefit from alL-6 antibody treatment and longterm benefits of 1-month treatment with alL-6 antibody. The combination of alL-6 antibody and rapamycin appears to have an additive effect on tumorigenesis. These studies support the therapeutic potential of using clinically available approaches to target IL-6 in patients with TSC and LAM alone or in combination with mTORC1 inhibition.

\section{Discussion}

We have discovered that IL-6 cooperates with mTORC1 activation to support TSCassociated metabolic reprogramming. Using steady state metabolomics in combination with $U-{ }^{13} \mathrm{C}$ glucose tracing we demonstrate that IL-6 plays a role in shunting glycolytic intermediates into de 
novo serine synthesis, a process which supports redox homeostasis and nucleotide metabolism in numerous tumors $(41,46-48)$. Our data suggest that IL-6 targeting has a significant impact on the metabolism and proliferation of TSC2-deficient cells. Interestingly, PSAT1 overexpression was sufficient to rescue the proliferation of the IL-6 knockout cells in the TSC2-deficient state, suggesting that regulation of serine metabolism is a key pathway downstream of IL-6. Serine, Glycine, and One Carbon (SGOC) metabolism fuels into numerous essential metabolic pathways $(48,49)$. Future studies will explore additional processes downstream of IL-6 and serine metabolism in the setting of mTORC1 hyperactivation. Of note, serine metabolism was shown to play a role in epigenetic processes following LKB1 loss and mTORC1 activation in KRAS-driven tumors (50). Serine availability is also important for the generation of certain lipid species, known to be dysregulated in TSC2-deficient cells, suggesting another aspect of TSC biology that may be significantly impacted by inhibiting IL-6 $(47,51)$. These studies highlight the far-reaching effects on features of TSC pathogenesis which may be mediated by IL-6 and serine metabolism.

IL-6 is a known autocrine, paracrine and endocrine factor that has been implicated in the initiation, progression and metastasis of numerous tumor types including skin, breast, lung, and kidney $(13,52,53)$. In this study we focused on the cell autonomous roles of IL-6 on the metabolism and tumorigenesis of TSC2-deficient cells. Interestingly, we discovered that circulating IL-6 levels are elevated in the serum of LAM patients, and in TSC2-deficient human angiomyolipoma cells. Additionally, expression of IL-6Ra is elevated in TSC2-deficient cells suggesting that IL-6 plays a role in a cell autonomous manner. Interestingly, IL-6Ra can be shed into the extracellular milieu, allowing previously non-responsive cells expressing the ubiquitous gp130 receptor to sense IL-6 $(42,54,55)$. This mechanism of action highlights the exciting possibility that TSC2-deficient cells may also shed IL-6R $\alpha$ to modulate the tumor microenvironment, exerting non-cell autonomous effects. Since recent studies have shown that TSC-associated pulmonary LAM and renal angiomyolipomas are responsive to immune checkpoint blockade $(28,56)$, the importance of the immune system in TSC and LAM disease progression has been well established. Importantly, IL-6 targeted therapies following mTORC1 
activation caused by STK11/LKB1 loss in RAS-driven lung tumors decreased the immunosuppressive effects of tumor associated neutrophils (57). Therefore, understanding the impact of IL-6 mediated signaling on the tumor microenvironment in TSC and LAM will be a critical next step for future studies.

IL-6 activates the pro-oncogenic transcription factor STAT3 via binding to IL-6Ra and gp130, leading to canonical JAK/STAT signaling. STAT3 activation is a well-described feature of TSC $(15,16,19,21,23,58,59)$, including both brain and lung manifestations (16, 20). However, the mechanisms underlying this activation are not completely understood. We propose a model in which secreted IL-6 potentiates the STAT3 signal in TSC2-deficient cells. STAT3 is also directly activated by mTORC1-mediated phosphorylation on serine 727 , a phosphorylation event required for maximal transcriptional activation (60). Together these data support the activation of a STAT3/IL-6 positive feedback loop in TSC (61). Surprisingly, we discovered that IL-6 regulates de novo serine synthesis in a STAT3-independent manner in TSC2-deficient cells. Interestingly, the transcription factor, ATF4, known to regulate de novo serine synthesis enzymes $(41,62)$, was both mTORC1-dependent and regulated by IL-6. IL-6 activates additional pro-tumorigenic pathways including RAS, phosphatidyl inositol 3-phosphate (PI3K-AKT), and Yes associated protein/taffazin (YAP/TAZ) (13, 63-65). Furthermore, the IL-6 gene promoter contains an antioxidant response element and can be regulated by Nuclear Factor Erythroid 2 (NRF2) (66), which is upregulated in TSC lesions (67), and has been previously implicated in regulating de novo serine synthesis in tumors (68). Finally, YAP/TAZ signaling has been shown to be upregulated in TSC and plays a role in the regulation of transaminases in cancers $(69,70)$. Our data suggest that the regulation of de novo serine synthesis downstream of IL-6 may involve a complex interplay of transcription factors, many of which are implicated in TSC and LAM pathogenesis.

This project highlights potential new therapeutic strategies for TSC and LAM. To assess the therapeutic potential of targeting IL-6 in TSC and LAM, we treated 8-month-old Tsc2 ${ }^{+/}$mice that have established tumor burden with IL-6 neutralizing antibody ( $\alpha$ IL-6 antibody). We 
demonstrate that alL-6 antibody suppresses the formation of renal cysts and cystadenomas in $T s c 2^{+/-}$mice. IL-6 targeted therapies are currently FDA-approved for the treatment of rheumatoid arthritis and Castleman's Disease $(54,55)$. Interestingly, we found only a partial reduction of IL-6 upon rapamycin treatment in vitro and an additive benefit of combining alL-6 antibody with rapamycin in vivo. These data suggest that IL-6 pathway targeted therapies may work well in combination with mTORC1 inhibition, which is standard of care for most patients with progressive TSC and LAM. Efforts are also underway to develop novel inhibitors of serine metabolism. These studies have shown that tumors must be in a serine limited environment for maximal therapeutic potential. The brain is one organ system with low serine and glycine in which PHGDH inhibitors have shown therapeutic benefit in preclinical models (71), with possible implications for the various brain tumors that form in TSC. Finally, a serine/glycine limited diet has also been shown to improve the efficacy of these inhibitors in tumor models $(72,73)$.

In summary, we have uncovered a distinct IL-6 dependent metabolic signature, which plays an important role in supporting the proliferative and bioenergetic activity of TSC2-deficient cells. In particular, IL-6 appears to promote de novo serine biosynthesis and expression of the key intermediate enzyme, PSAT1. Targeting IL-6 with a neutralizing antibody exerts acute and durable therapeutic responses alone and in combination with rapamycin in vivo. Future studies may elucidate the molecular mechanisms by which IL-6 regulates de novo serine biosynthesis and the potential therapeutic benefits of directly targeting de novo serine metabolism in TSC and LAM.

\section{Materials and Methods}

Cell lines and treatment. $T s c 2^{-/ p} p 53^{-/}$and $T s c 2^{+/+} p 53^{-/-}$mouse embryonic fibroblasts (MEF) were provided by David Kwiatkowski (Brigham and Women's Hospital, Boston, MA). The MEFs and stocks prepared at passage 8 . TTJ cells and $105 \mathrm{~K}$ were derived from a renal cystadenoma of a C57BL/6 $\mathrm{Tsc}^{+/-}$mouse, with re-expressed Tsc2 or empty vector, as previously described (28). 
The 621-101 cells were isolated from a patient renal angiomyolipoma and immortalized with E6/E7, and the parental 621-101 cells were then use to re-express Tsc2 (621-103) or empty vector (621-102), as previously described $(74,75)$. HEK293 cells were obtained from ATCC. The inducible raptor and rictor knockout MEFs were generated in the laboratory of Michael Hall, the cells were cultured in $10 \%$ FBS in DMEM and treated with $1 \mathrm{uM}$ of tamoxifen or ethanol control for $72 \mathrm{~h}$ to induce knockout prior to harvest (40). For IL-6 knockout, TSC2-deficient cells were transduced with single lentivirus containing an spCas9 and sgRNA (CTTCCCTACTTCACAAGTC) expression cassette to target spCas9 cleavage to IL-6. The lentiviral plasmids (LV01) and lentivirus production were obtained from Sigma-Aldrich. Cells were sorted for GFP expression by flow cytometry and maintained in puromycin $(3 \mu \mathrm{g} / \mathrm{mL})$. IL-6 knockout was confirmed by Sanger sequencing and ELISA. For PSAT1 overexpression, lentiviral vector of PSAT1 (EX-Mm13089-Lv197-GS, GeneCopoeia) was transfected into HEK-293T cells along with lentiviral packaging mix to produce lentivirus. Tsc2-deficient cells with or without IL-6 overexpression were transfected with lentivirus and selected with blasticidin at $10 \mu \mathrm{g} / \mathrm{ml}$. Knockdown experiments were performed using Silencer Select siRNA from Ambion STAT3 (4392420) and IL-6RAa (4390771) transfected using Lipofectamine RNAiMax Reagent (Invitrogen). All cells tested negative for mycoplasma contamination using MycoAlert (Lonza) and were re-tested monthly. Cells were cultured at $37^{\circ} \mathrm{C}$ in $5 \% \mathrm{CO} 2$ in DMEM supplemented with $10 \%$ FBS and gentamycin sulfate $(50 \mu \mathrm{g} / \mathrm{mL})$. For serum-free conditions, cells were cultured in DMEM without serum.

Antibodies and drugs. The following antibodies were used: TSC2 (Cell Signaling Technology, 4308S), S6 (2217S), pS6(2211L), IL-6 (Santa Cruz Biotechnology, sc-57315), STAT3 (9139S), pSTAT3 (9145S), PSAT1 (Protein Tech, \#20180-1-AP), PHGDH (Protein Tech, 14719-1-AP), PSPH (Protein Tech, 14513-1-AP), ß-actin (Sigma-Aldrich), Ki-67 (ebioscience, 14-5698-82). For immunohistochemistry, PSAT1 antibody (2102) was purchased form Origene. IL-6 neutralizing 
antibody and IgG control (Bioxcell, BE0046 and BE0088). Rapamycin and Torin1 were purchased from LC Laboratories. Recombinant mouse IL-6 (406-ML) was purchased from R\&D.

Seahorse assay. The MitoStress Test Assay and the Seahorse XFe24 analyzer were used. Cells were seeded into the XFe24 microplate and incubated for 24 hours in 10\% FBS DMEM. The next day, cells were washed by PBS and cultured in FBS free DMEM for 24 hours. Add compounds (final concentrations: $1 \mu \mathrm{M}$ oligomycin, $1 \mu \mathrm{M}$ FCCP, $0.5 \mu \mathrm{M}$ rotenone/antimycin $\mathrm{A}$ ) to pre-hydrated sensor cartridge. The sensor cartridge and XFe 24 microplate were placed in the XFe Analyzer. Results were normalized to cell number.

Cytokine Array. A RayBiotech Human Cytokine Array 5 was used to detect differential secretion of cytokines from angiomyolipoma-derived 621-101 cells compared to human embryonic kidney HEK293 cells according to the manufacturer's instructions. Cells were grown in IIA Complete Media and serum starved overnight (16h) in $3 \mathrm{ml}$ of media on $10 \mathrm{~cm}$ dishes. One $\mathrm{mL}$ of media was applied to the cytokine array and incubated for 2 hours. As a control, one array was incubated with unconditioned media.

ELISA. ELISA was performed using conditioned media ( $\sim 80 \%$ confluent cells, concentrated with Millipore UFC 900324 filters) and normalized by protein concentration (Bio-Rad Laboratories, \#5000006). Levels of secreted IL-6 were determined according to the manufacturer's protocol (R\&D Systems, IL-6 Quantikine ELISA Kit).

Crystal violet assay. Cells were seeded at a density of 1,000 cells/well in 96-well plates and changed into serum-free media after 24 hours. At the indicated time points, cells were fixed for 15 minutes with $10 \%$ formalin and then stained with $0.5 \%$ crystal violet in distilled water for 20 minutes. Crystal violet was removed and cells were washed with water followed by drying at room temperature. Crystal violet was solubilized with $200 \mathrm{ml}$ of methanol and measured with a plate 
reader (OD 540; BioTek, Winooski, VT, USA). Proliferation was assessed by comparing the change in OD 540 at 24,48 and $72 \mathrm{~h}$ as normalized to $0 \mathrm{~h}$ (start of serum-free proliferation) for each cell line.

Transwell migration assay. Migration was evaluated as described previously (76). Briefly $5 \times 10^{4}$ cells were seeded in $100 \mu \mathrm{l}$ of serum-free DMEM in the upper chamber of a $6.5 \mathrm{~mm}$ polycarbonate Transwell with $8.0 \mu \mathrm{m}$ pores (Corning, USA), through which the cells were allowed to migrate for $6 \mathrm{~h}$ at $37^{\circ} \mathrm{C}$ toward $10 \%$ FBS in the basal compartment. At the end of the incubation, migrated cells on the lower surface of the transwell were fixed, stained, and quantified.

Soft agar assay. Soft agar assays were performed to measure anchorage-independent growth. Briefly, $5 \times 10^{3}$ cells were placed into a single well in a 6 -well plate. Cells were embedded into $0.4 \%$ low-melting agarose (Sigma) and layered on top of a $0.8 \%$ agarose base. After 2 weeks of growth, the cells were fixed and analyzed. Colony number was quantitated using ImageJ (v1.53).

Wound healing assay. Cells were seeded into six-well plates in DMEM culture medium and allowed to grow for 24 hours until confluency was reached. Cells were then washed with 1x PBS, and a scratch was made using a $200 \mu$ lip at the center of the well. The monolayers were imaged at the indicated times using a light microscope at 100x magnification. The results were quantified using ImageJ (v1.53) software.

RNA extraction and quantitative real-time PCR. Total RNA was extracted using RNeasy Mini Kit (QIAGEN, USA). The RNA concentration was measured using a Nanodrop 2000c (Thermo Scientific, USA). Two micrograms of RNA were reverse transcribed using a High-Capacity cDNA Reverse Transcription Kit (Thermofisher, USA) with random primers. For qPCR, a Taqman-based method was used and the relative quantitation of gene expression was determined using the 
comparative CT $(\triangle \triangle \mathrm{CT})$ method and normalized to $\beta$-actin gene and a calibrator sample that was run on the same plate. PCR primers and probe sets were obtained from Thermofisher, USA: IL-6 (assay ID Mm00446191_m1, 124 bp amplicon length), PSAT1 (assay ID Mm04932904_m1, 109 bp amplicon length), and $\beta$-actin control (cat\# 4351315).

Western blot analysis. After indicated treatment, live cells were lysed on ice in 1× RIPA (Cell Signaling Technology) containing phosphatase and protease inhibitors. For the membrane fractionation experiments Mem-PER ${ }^{\mathrm{TM}}$ Plus Membrane Protein Extraction Kit was used according to manufacturer's instructions (Thermo Scientific, 89842). The concentration of proteins was determined using a Bio-Rad Protein Assay Dye Reagent Concentrate (Bio-Rad Laboratories, \#5000006). A total of $15 \mu \mathrm{g}$ of protein from each sample were mixed with NuPAGE ${ }^{\text {TM }}$ LDS Sample Buffer (Thermofisher, NP0007) and Reducing Sample Buffer (Invitrogen, NP009, USA), resolved on a $4-12 \%$ Bis-Tris gels (Thermofisher), then transferred to PVDF membranes (MilliporeSigma, USA). Blots were blocked with $5 \%$ milk and incubated with primary and second antibodies. Chemiluminescence was captured with Syngene G-Box gel documentation system.

Immunohistochemistry staining. Kidneys were formalin-fixed, paraffin-embedded, and tissue was cut in 3- to 4- $\mu \mathrm{m}$ sections then air-dried overnight. The sections were deparaffinized, rehydrated, and subjected to heat-induced epitope retrieval using low $\mathrm{pH}$ target retrieval solution for 15 minutes. Sections were incubated with Ki67 of PSAT1 primary antibody (1:100 dilution). Slides were developed using DAB and counterstained with hematoxylin.

Targeted Mass Spectrometry. Samples were re-suspended using $20 \mathrm{~mL}$ HPLC grade water for mass spectrometry. 5-7 $\mu \mathrm{L}$ were injected and analyzed using a hybrid 6500 QTRAP triple quadrupole mass spectrometer (AB/SCIEX) coupled to a Prominence UFLC HPLC system (Shimadzu) via selected reaction monitoring (SRM) of a total of 270 endogenous water-soluble metabolites for steady-state analyses of samples. Some metabolites were targeted in both 
positive and negative ion mode for a total of 305 SRM transitions using positive/negative ion polarity switching. ESI voltage was $+4950 \mathrm{~V}$ in positive ion mode and $-4500 \mathrm{~V}$ in negative ion mode. The dwell time was $3 \mathrm{~ms}$ per SRM transition and the total cycle time was 1.39 seconds. Approximately 10-14 data points were acquired per detected metabolite. Samples were delivered to the mass spectrometer via hydrophilic interaction chromatography (HILIC) using a $4.6 \mathrm{~mm}$ i.d x $10 \mathrm{~cm}$ Amide XBridge column (Waters) at $400 \mu \mathrm{L} / \mathrm{min}$. Gradients were run starting from $85 \%$ buffer B (HPLC grade acetonitrile) to $42 \%$ B from $0-5$ minutes; $42 \%$ B to $0 \%$ B from 5 16 minutes; 0\% B was held from 16-24 minutes; 0\% B to $85 \%$ B from $24-25$ minutes; $85 \%$ B was held for 7 minutes to re-equilibrate the column. Buffer A was comprised of $20 \mathrm{mM}$ ammonium hydroxide/20 mM ammonium acetate $(\mathrm{pH}=9.0)$ in 95:5 water:acetonitrile. Peak areas from the total ion current for each metabolite SRM transition were integrated using MultiQuant v3.0 software $(A B / S C I E X)(36,39)$. Analysis of metabolomics data including the generation of heatmaps and metabolite set enrichment analysis (MSEA) was performed using the open access MetaboAnalyst Software (v4.0 or 5.0).

U- ${ }^{13}$ C Glucose Tracing. Cells $\left(4 \times 10^{5}\right)$ were seeded onto $60 \mathrm{~mm}$ plates in $10 \%$ FBS DMEM (ThermoFisher Scientific, Gibco \#11995123). The next day the cells were washed with PBS and transferred to serum-free DMEM (Thermo Fisher Scientific, Gibco \#11966025) supplemented with $4.5 \mathrm{~g} / \mathrm{L}$ D-glucose. For U-13 C Glucose Tracing following alL-6 antibody experiments, the cells also were washed with PBS and transferred to either alL- 6 antibody $(10 \mathrm{ug} / \mathrm{ml})$ or $\operatorname{lgG}(10 \mathrm{ug} / \mathrm{ml})$ in serum-free DMEM (Thermo Fisher Scientific, Gibco \#11966025) supplemented with $4.5 \mathrm{~g} / \mathrm{L}$ Dglucose. At $-24 h,-3 h,-1 h$ to harvest, the cells were washed with glucose-free and serum-free DMEM (Gibco \#11966025) and then incubated in DMEM (\#11966-025) supplemented with 4.5 $\mathrm{g} / \mathrm{L} \mathrm{U}-{ }^{13} \mathrm{C}$ Glucose (Sigma Aldrich) for $0 \mathrm{~h}, 1 \mathrm{~h}, 3 \mathrm{~h}$, or $24 \mathrm{~h}$. The cells were harvested and analyzed as described above $(36,39)$. 
Human plasma specimens. Patient samples and healthy control samples were obtained through a Partner's Health Care Institutional Review Board approved protocol. Subjects were consented by the clinical research team free of coercion and samples were collected, deidentified, and coded. Plasma Interleukin-6 was measured by ELISA (R\&D Quantikine Human IL-6 ELISA or High Sensitivity IL-6 ELISA).

Animal studies. All animal studies were performed in accordance with institutional protocols approved by BWH Institutional Animal Care and Use Committee. For the cystic kidney model, we crossed the CAGGCRE-ER ${ }^{\mathrm{TM}+/}$ (The Jackson Laboratory) to Tsc2 flox/flox (Michael Gambello). Recombination of Tsc2 was induced in 8-10 week old CAGGCRE-ER ${ }^{\mathrm{TM}+/-} ; \mathrm{Tsc}^{\text {flox/flox }}$ or controls (CAGGCRE-ER ${ }^{\mathrm{TM}-1-\text {; }}$ Tsc2 ${ }^{\text {flox/flox }}$ ) mice with intraperitoneal tamoxifen (in corn oil) at a dose of $1 \mathrm{mg}$ per day for 5 consecutive days. Kidneys were harvested at 5 months of age. $\mathrm{Tsc}^{+/-}$mice in the $\mathrm{A} / \mathrm{J}$ background were generated in house as described previously $(43,77)$. Intraperitoneal injection was done with 200 ug of $\alpha \mathrm{lL}-6$ antibody or control rat IgG antibody (Bioxcell) three times per week as previously described (57). After a total of 4 weeks of treatment, mice were harvested and the severity of renal lesions was scored using previously established macroscopic and microscopic scoring methods $(43,77)$.Macroscopic cysts per kidney were scored according to size: $<1 \mathrm{~mm}$, score $1 ; 1-1.5$, score $2 ; 1.5-2$, score 5 ; and $>2$, score 10 . The sum of the cyst scores were determined and reported per kidney. Microscopic kidney tumor scores were determined by an observer blinded to the experimental conditions using a semi-quantitative algorithm and hematoxylin and eosin (H\&E) sections of samples prepared by embedding $1 \mathrm{~mm}$ interval sections. Each tumor or cyst identified was measured (length, width) and percent of the lumen filled by tumor determined ( $0 \%$ for a simple cyst, and $100 \%$ for a completely filled, solid tumor). The measurements were converted into a score using a previously established formula (43). Semi-quantitative analysis of PSAT1 immunohistochemistry was performed by a blinded observer who scored the PSAT1 signal on a sale from 0-5 (0-no signal, 5-maximum observed signal) in $\sim 10$ renal lesions per mouse. The average score for each mouse was then calculated 
and the Log2 fold change of the alL-6 antibody treated mice was calculated relative to the IgG control mice.

Statistical analyses. Normally distributed data were analyzed for statistical significance with Student's unpaired t-test and multiple comparisons were made with One-Way and Two-Way ANOVAs with Bonferroni correction. In vivo data are presented as the mean $+/-95 \%$ confidence interval $(\mathrm{Cl})$ and in vitro studies are presented as the mean $+/-$ standard deviation (SD). Analysis was performed using GraphPad Prism version 8; GraphPad Software, www.graphpad.com. Statistical significance was defined as $p<0.05$.

\section{Acknowledgments}

This work was funded by the NIH (K01-DK116819 to HCL, R01HL146541 to WS, U01 HL13102204 to $\mathrm{EPH}$, ) and The Engles Family TSC/LAM Research Fund. The mass spectrometry work was partially funded by NIH grants 5P01CA120964 (J.M.A.) and 5P30CA006516 (J.M.A.). We thank Dana-Farber/Harvard Cancer Center in Boston, MA for the use of the Rodent Histopahtology Core, which provided tissue embedding and sectioning services. Dana-Farber/Harvard Cancer Center is supported in part by a NCI Cancer Center Support Grant \# NIH 5P30 CA06516. We would also like to acknowledge Clemens K. Probst for technical assistance with experiments. Schematics were generated using Biorender.com. This work was performed in part to meet the requirements of the doctoral thesis of Dr. Ji Wang from Zhejiang University School of Medicine, Hangzhou, China. 


\section{References}

1. J. P. Osborne, A. Fryer, D. Webb, Epidemiology of tuberous sclerosis. Ann N Y Acad Sci 615, 125-127 (1991).

2. F. J. O'Callaghan, A. W. Shiell, J. P. Osborne, C. N. Martyn, Prevalence of tuberous sclerosis estimated by capture-recapture analysis. Lancet 351, 1490 (1998).

3. L. Hallett, T. Foster, Z. Liu, M. Blieden, J. Valentim, Burden of disease and unmet needs in tuberous sclerosis complex with neurological manifestations: systematic review. Curr Med Res Opin 27, 1571-1583 (2011).

4. E. P. Henske, S. Jozwiak, J. C. Kingswood, J. R. Sampson, E. A. Thiele, Tuberous sclerosis complex. Nat Rev Dis Primers 2, 16035 (2016).

5. C. W. Shepherd, M. R. Gomez, J. T. Lie, C. S. Crowson, Causes of death in patients with tuberous sclerosis. Mayo Clin Proc 66, 792-796 (1991).

6. H. Northrup, D. A. Krueger, G. International Tuberous Sclerosis Complex Consensus, Tuberous sclerosis complex diagnostic criteria update: recommendations of the 2012 linternational Tuberous Sclerosis Complex Consensus Conference. Pediatr Neurol 49, 243-254 (2013).

7. H. C. Lam, B. J. Siroky, E. P. Henske, Renal disease in tuberous sclerosis complex: pathogenesis and therapy. Nat Rev Nephrol 14, 704-716 (2018).

8. K. H. Yu et al., Data-driven analyses revealed the comorbidity landscape of tuberous sclerosis complex. Neurology 91, 974-976 (2018).

9. A. Peron, K. S. Au, H. Northrup, Genetics, genomics, and genotype-phenotype correlations of TSC: Insights for clinical practice. Am J Med Genet C Semin Med Genet 178, 281-290 (2018).

10. I. Ben-Sahra, B. D. Manning, mTORC1 signaling and the metabolic control of cell growth. Curr Opin Cell Biol 45, 72-82 (2017).

11. G. Y. Liu, D. M. Sabatini, mTOR at the nexus of nutrition, growth, ageing and disease. Nat Rev Mol Cell Biol 10.1038/s41580-019-0199-y (2020).

12. L. J. McEneaney, A. R. Tee, Finding a cure for tuberous sclerosis complex: From genetics through to targeted drug therapies. Adv Genet 103, 91-118 (2019).

13. K. Taniguchi, M. Karin, IL-6 and related cytokines as the critical lynchpins between inflammation and cancer. Semin Immunol 26, 54-74 (2014).

14. D. Iliopoulos, H. A. Hirsch, K. Struhl, An epigenetic switch involving NF-kappaB, Lin28, Let7 MicroRNA, and IL6 links inflammation to cell transformation. Cell 139, 693-706 (2009).

15. K. M. Dodd, J. Yang, M. H. Shen, J. R. Sampson, A. R. Tee, mTORC1 drives HIF-1alpha and VEGF-A signalling via multiple mechanisms involving 4E-BP1, S6K1 and STAT3. Oncogene 34, 2239-2250 (2015).

16. E. A. Goncharova et al., Signal transducer and activator of transcription 3 is required for abnormal proliferation and survival of TSC2-deficient cells: relevance to pulmonary lymphangioleiomyomatosis. Mol Pharmacol 76, 766-777 (2009).

17. N. El-Hashemite, D. J. Kwiatkowski, Interferon-gamma-Jak-Stat signaling in pulmonary lymphangioleiomyomatosis and renal angiomyolipoma: a potential therapeutic target. $\mathrm{Am}$ J Respir Cell Mol Biol 33, 227-230 (2005).

18. J. A. Chan et al., Pathogenesis of tuberous sclerosis subependymal giant cell astrocytomas: biallelic inactivation of TSC1 or TSC2 leads to mTOR activation. $J$ Neuropathol Exp Neurol 63, 1236-1242 (2004).

19. N. El-Hashemite, H. Zhang, V. Walker, K. M. Hoffmeister, D. J. Kwiatkowski, Perturbed IFN-gamma-Jak-signal transducers and activators of transcription signaling in tuberous sclerosis mouse models: synergistic effects of rapamycin-IFN-gamma treatment. Cancer Res 64, 3436-3443 (2004).

20. H. Onda et al., Tsc2 null murine neuroepithelial cells are a model for human tuber giant cells, and show activation of an mTOR pathway. Mol Cell Neurosci 21, 561-574 (2002). 
21. E. N. Atochina-Vasserman et al., Surfactant dysfunction and lung inflammation in the female mouse model of lymphangioleiomyomatosis. Am J Respir Cell Mol Biol 53, 96-104 (2015).

22. E. A. Goncharova et al., Prevention of alveolar destruction and airspace enlargement in a mouse model of pulmonary lymphangioleiomyomatosis (LAM). Sci Transl Med 4, 154ra134 (2012).

23. E. Lesma et al., TSC2 epigenetic defect in primary LAM cells. Evidence of an anchorageindependent survival. J Cell Mol Med 18, 766-779 (2014).

24. K. R. Mattaini, M. R. Sullivan, M. G. Vander Heiden, The importance of serine metabolism in cancer. J Cell Biol 214, 249-257 (2016).

25. M. Reina-Campos, M. T. Diaz-Meco, J. Moscat, The complexity of the serine glycine onecarbon pathway in cancer. J Cell Biol 219 (2020).

26. M. Ding, R. K. Bruick, Y. Yu, Secreted IGFBP5 mediates mTORC1-dependent feedback inhibition of IGF-1 signalling. Nat Cell Biol 18, 319-327 (2016).

27. H. Zhang et al., Loss of Tsc1/Tsc2 activates mTOR and disrupts PI3K-Akt signaling through downregulation of PDGFR. J Clin Invest 112, 1223-1233 (2003).

28. H. J. Liu et al., TSC2-deficient tumors have evidence of T cell exhaustion and respond to anti-PD-1/anti-CTLA-4 immunotherapy. JCl Insight 3 (2018).

29. H. C. Lam et al., p62/SQSTM1 Cooperates with Hyperactive mTORC1 to Regulate Glutathione Production, Maintain Mitochondrial Integrity, and Promote Tumorigenesis. Cancer Res 77, 3255-3267 (2017).

30. N. Liang et al., Regulation of YAP by mTOR and autophagy reveals a therapeutic target of tuberous sclerosis complex. J Exp Med 211, 2249-2263 (2014).

31. F. X. McCormack, W. D. Travis, T. V. Colby, E. P. Henske, J. Moss, Lymphangioleiomyomatosis: calling it what it is: a low-grade, destructive, metastasizing neoplasm. Am J Respir Crit Care Med 186, 1210-1212 (2012).

32. M. Guo et al., Single-Cell Transcriptomic Analysis Identifies a Unique Pulmonary Lymphangioleiomyomatosis Cell. Am J Respir Crit Care Med 202, 1373-1387 (2020).

33. H. C. Lam et al., Rapamycin-induced miR-21 promotes mitochondrial homeostasis and adaptation in mTORC1 activated cells. Oncotarget 8, 64714-64727 (2017).

34. C. Priolo, E. P. Henske, Metabolic reprogramming in polycystic kidney disease. Nat Med 19, 407-409 (2013).

35. E. E. Verwer et al., [(18)F]Fluorocholine and [(18)F]Fluoroacetate PET as Imaging Biomarkers to Assess Phosphatidylcholine and Mitochondrial Metabolism in Preclinical Models of TSC and LAM. Clin Cancer Res 24, 5925-5938 (2018).

36. M. Yuan, S. B. Breitkopf, X. Yang, J. M. Asara, A positive/negative ion-switching, targeted mass spectrometry-based metabolomics platform for bodily fluids, cells, and fresh and fixed tissue. Nat Protoc 7, 872-881 (2012).

37. M. A. Hawk, Z. T. Schafer, Mechanisms of redox metabolism and cancer cell survival during extracellular matrix detachment. J Biol Chem 293, 7531-7537 (2018).

38. R. Possemato et al., Functional genomics reveal that the serine synthesis pathway is essential in breast cancer. Nature 476, 346-350 (2011).

39. M. Yuan et al., Ex vivo and in vivo stable isotope labelling of central carbon metabolism and related pathways with analysis by LC-MS/MS. Nat Protoc 14, 313-330 (2019).

40. N. Cybulski, V. Zinzalla, M. N. Hall, Inducible raptor and rictor knockout mouse embryonic fibroblasts. Methods Mol Biol 821, 267-278 (2012).

41. I. Ben-Sahra, G. Hoxhaj, S. J. H. Ricoult, J. M. Asara, B. D. Manning, mTORC1 induces purine synthesis through control of the mitochondrial tetrahydrofolate cycle. Science $\mathbf{3 5 1}$, 728-733 (2016).

42. E. H. Choy et al., Translating IL-6 biology into effective treatments. Nat Rev Rheumatol 16, 335-345 (2020).

43. L. Lee et al., Efficacy of a rapamycin analog (CCl-779) and IFN-gamma in tuberous sclerosis mouse models. Genes Chromosomes Cancer 42, 213-227 (2005). 
44. C. D. Burger, Efficacy and safety of sirolimus in lymphangioleiomyomatosis. N Engl J Med 365, 271-272; author reply 272 (2011).

45. J. J. Bissler et al., Sirolimus for angiomyolipoma in tuberous sclerosis complex or lymphangioleiomyomatosis. N Engl J Med 358, 140-151 (2008).

46. I. Ben-Sahra, J. J. Howell, J. M. Asara, B. D. Manning, Stimulation of de novo pyrimidine synthesis by growth signaling through mTOR and S6K1. Science 339, 1323-1328 (2013).

47. X. Gao et al., Serine Availability Influences Mitochondrial Dynamics and Function through Lipid Metabolism. Cell Rep 22, 3507-3520 (2018).

48. M. Mehrmohamadi, X. Liu, A. A. Shestov, J. W. Locasale, Characterization of the usage of the serine metabolic network in human cancer. Cell Rep 9, 1507-1519 (2014).

49. M. A. Reid et al., Serine synthesis through PHGDH coordinates nucleotide levels by maintaining central carbon metabolism. Nat Commun 9, 5442 (2018).

50. F. Kottakis et al., LKB1 loss links serine metabolism to DNA methylation and tumorigenesis. Nature 539, 390-395 (2016).

51. C. Priolo et al., Tuberous sclerosis complex 2 loss increases lysophosphatidylcholine synthesis in lymphangioleiomyomatosis. Am J Respir Cell Mol Biol 53, 33-41 (2015).

52. Y. Zhu et al., p21-activated kinase 1 determines stem-like phenotype and sunitinib resistance via NF-kappaB/IL-6 activation in renal cell carcinoma. Cell Death Dis 6, e1637 (2015).

53. T. Cuadros et al., HAVCR/KIM-1 activates the IL-6/STAT-3 pathway in clear cell renal cell carcinoma and determines tumor progression and patient outcome. Cancer Res 74, 14161428 (2014).

54. C. Garbers, S. Heink, T. Korn, S. Rose-John, Interleukin-6: designing specific therapeutics for a complex cytokine. Nat Rev Drug Discov 17, 395-412 (2018).

55. T. Tanaka, T. Kishimoto, The biology and medical implications of interleukin-6. Cancer Immunol Res 2, 288-294 (2014).

56. H. J. Liu, V. P. Krymskaya, E. P. Henske, Immunotherapy for Lymphangioleiomyomatosis and Tuberous Sclerosis: Progress and Future Directions. Chest 156, 1062-1067 (2019).

57. S. Koyama et al., STK11/LKB1 Deficiency Promotes Neutrophil Recruitment and Proinflammatory Cytokine Production to Suppress T-cell Activity in the Lung Tumor Microenvironment. Cancer Res 76, 999-1008 (2016).

58. J. Ma et al., Mammalian target of rapamycin regulates murine and human cell differentiation through STAT3/p63/Jagged/Notch cascade. J Clin Invest 120, 103-114 (2010).

59. Y. Cui et al., Aberrant SYK Kinase Signaling Is Essential for Tumorigenesis Induced by TSC2 Inactivation. Cancer Res 77, 1492-1502 (2017).

60. K. Yokogami, S. Wakisaka, J. Avruch, S. A. Reeves, Serine phosphorylation and maximal activation of STAT3 during CNTF signaling is mediated by the rapamycin target mTOR. Curr Biol 10, 47-50 (2000).

61. E. Rad, K. Dodd, L. Thomas, M. Upadhyaya, A. Tee, STAT3 and HIF1alpha Signaling Drives Oncogenic Cellular Phenotypes in Malignant Peripheral Nerve Sheath Tumors. Mol Cancer Res 13, 1149-1160 (2015).

62. J. Ye et al., Pyruvate kinase M2 promotes de novo serine synthesis to sustain mTORC1 activity and cell proliferation. Proc Natl Acad Sci U S A 109, 6904-6909 (2012).

63. K. Taniguchi et al., A gp130-Src-YAP module links inflammation to epithelial regeneration. Nature 519, 57-62 (2015).

64. K. Taniguchi et al., YAP-IL-6ST autoregulatory loop activated on APC loss controls colonic tumorigenesis. Proc Natl Acad Sci U S A 114, 1643-1648 (2017).

65. W. J. Azar et al., Noncanonical IL6 Signaling-Mediated Activation of YAP Regulates Cell Migration and Invasion in Ovarian Clear Cell Cancer. Cancer Res 80, 4960-4971 (2020).

66. C. J. Wruck et al., Nrf2 induces interleukin-6 (IL-6) expression via an antioxidant response element within the IL-6 promoter. J Biol Chem 286, 4493-4499 (2011).

67. M. Zarei et al., Tumors with TSC mutations are sensitive to CDK7 inhibition through NRF2 and glutathione depletion. J Exp Med 216, 2635-2652 (2019). 
68. G. M. DeNicola et al., NRF2 regulates serine biosynthesis in non-small cell lung cancer. Nat Genet 47, 1475-1481 (2015).

69. N. Liang, M. Pende, YAP enters the mTOR pathway to promote tuberous sclerosis complex. Mol Cell Oncol 2, e998100 (2015).

70. C. S. Yang et al., Glutamine-utilizing transaminases are a metabolic vulnerability of TAZ/YAP-activated cancer cells. EMBO Rep 19 (2018).

71. B. Ngo et al., Limited Environmental Serine and Glycine Confer Brain Metastasis Sensitivity to PHGDH Inhibition. Cancer Discov 10, 1352-1373 (2020).

72. O. D. Maddocks et al., Serine starvation induces stress and p53-dependent metabolic remodelling in cancer cells. Nature 493, 542-546 (2013).

73. S. C. Baksh et al., Extracellular serine controls epidermal stem cell fate and tumour initiation. Nat Cell Biol 22, 779-790 (2020).

74. J. Yu, A. Astrinidis, E. P. Henske, Chromosome 16 loss of heterozygosity in tuberous sclerosis and sporadic lymphangiomyomatosis. Am J Respir Crit Care Med 164, 15371540 (2001).

75. J. Yu, A. Astrinidis, S. Howard, E. P. Henske, Estradiol and tamoxifen stimulate LAMassociated angiomyolipoma cell growth and activate both genomic and nongenomic signaling pathways. Am J Physiol Lung Cell Mol Physiol 286, L694-700 (2004).

76. H. J. Liu et al., Rapamycin-upregulated miR-29b promotes mTORC1-hyperactive cell growth in TSC2-deficient cells by downregulating tumor suppressor retinoic acid receptor beta (RARbeta). Oncogene 38, 7367-7383 (2019).

77. K. Pollizzi, I. Malinowska-Kolodziej, M. Stumm, H. Lane, D. Kwiatkowski, Equivalent benefit of mTORC1 blockade and combined PI3K-mTOR blockade in a mouse model of tuberous sclerosis. Mol Cancer 8, 38 (2009). 
A
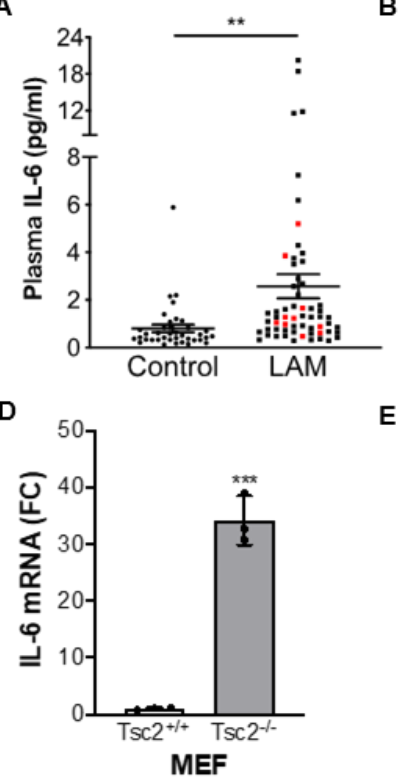

B

E
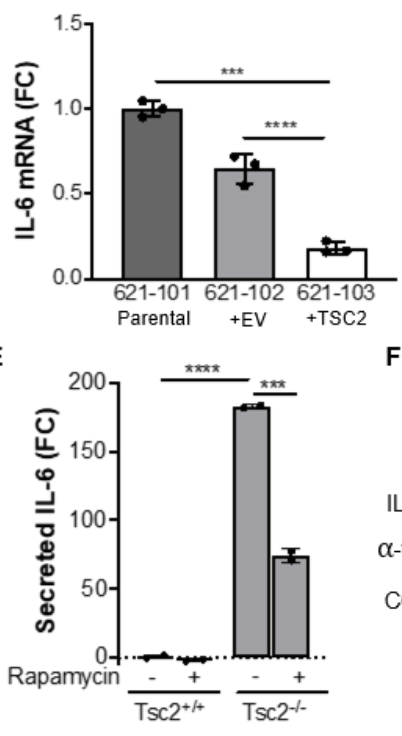

C

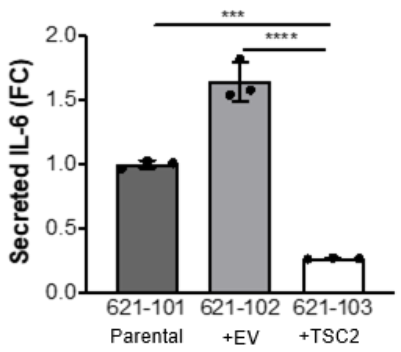

$\mathbf{F}$

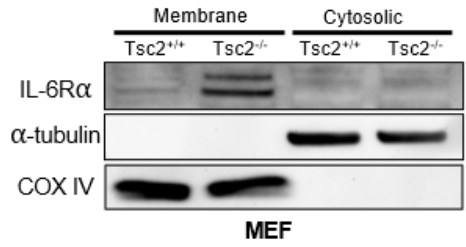

G

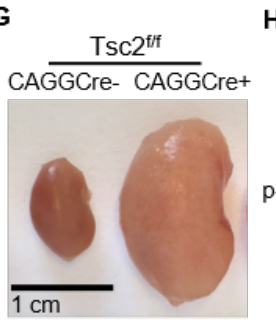

$\mathbf{H}$

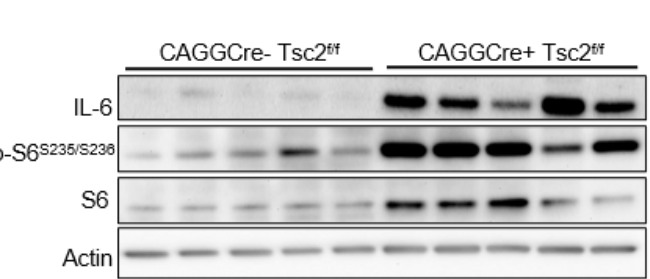

Figure 1. IL-6 is overexpressed in TSC2-deficient cells and tissues. (A) IL-6 was increased in the plasma of LAM patients (total $n=60$, black dots: sporadic-LAM, $n=50$; red dots: TSC-LAM, $n=10)$ compared to healthy control $(n=38)$. The data are presented as the mean \pm SEM. $(B)$ IL-6 mRNA expression by qRT-PCR and $(C)$ secreted IL-6 measured by ELISA are increased in TSC2-deficient human angiomyolipoma parental cells (621-101) and cells expressing empty vector (621-102), compared to cells with TSC2 addback (621-103). (D) qRT-PCR of IL-6 mRNA expression showing a 30-fold increase in Tsc2 ${ }^{-1-}$ MEFs compared to Tsc2 ${ }^{+/}$MEFs. $(E)$ Rapamycin treatment decreases secreted IL-6 in TSC2-deficient MEFs as measured by ELISA, (rapamycin; 20nM, 24 hours). (F) Western blot of membrane and cytosolic protein fractions of TSC2-deficient and wildtype MEFs. IL-6 receptor (IL-6Ra) is highly expressed in TSC2-deficient MEFs compared to TSC2-expressing MEFs. Cells were cultured in serum-free DMEM for 24 hours before harvesting. $\alpha$-tubulin used as a cytosolic fraction marker and COX IV as a membrane fraction marker. $(G)$ Representative kidneys of CAGGCre-ER $R^{T++} ; T s c 2^{\text {fff }}$ and


$E R^{T M--} ; T s c 2^{\text {fff }}$ kidneys showing increased IL-6 expression upon TSC2 loss. (I) Densitometry of IL-6 protein levels normalized to actin in CAGGCre-ER ${ }^{T M+-} ;$ Tsc2 $2^{f f f}$ and CAGGCre-ER $R^{T M-/} ; T s c 2^{f f f}$ kidney lysates shown in $H$. Data are presented as the mean $\pm \mathrm{SD}$ of three independent experiments, unless indicated otherwise. One-Way ANOVA, Two-Way ANOVA, or Student's t test were used for statistical analysis. ${ }^{*} p<0.05,{ }^{* \star} p<0.01,{ }^{* \star *} p<0.001,{ }^{* \star \star *} p<0.0001$. 
A



c
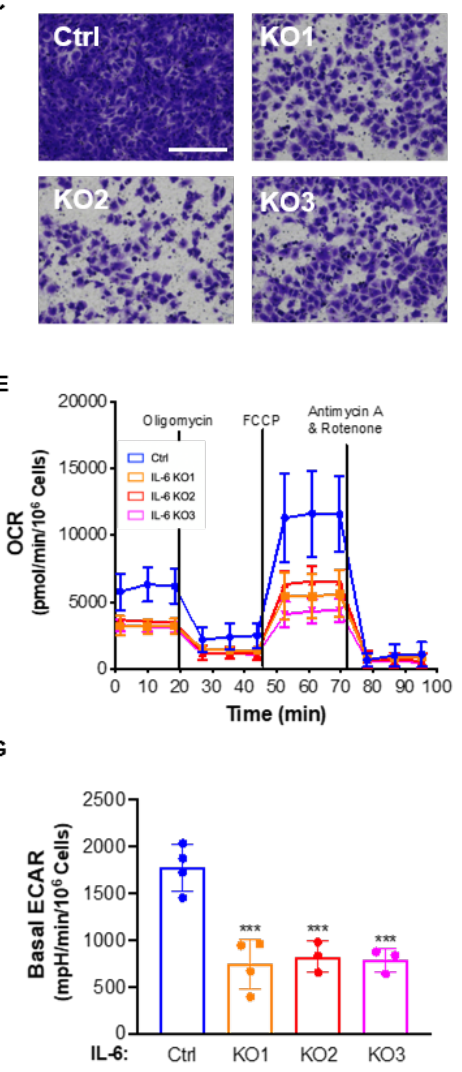

B

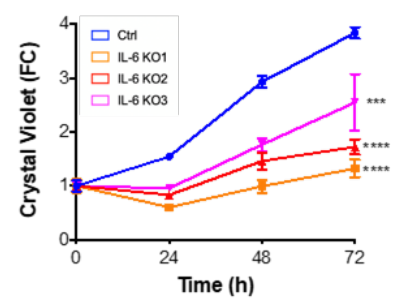

D
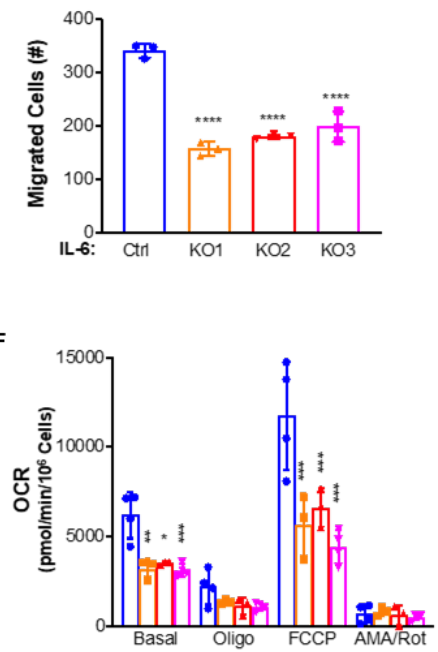

H

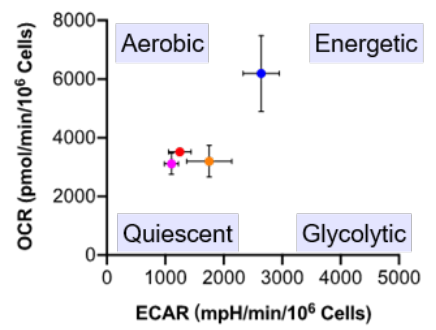

Figure 2. IL-6 knockout suppresses proliferation, migration, and induces a metabolic quiescent state in TSC2-deficient cells. ( $A$ ) Secreted IL-6 is decreased in three IL-6 CRISPR/Cas9 clones compared to control, as measured by ELISA. (B) IL-6 knockout decreases the proliferation of TSC2-deficient MEFs compared to control as measured by crystal violet staining as a readout of cell density. $(C)$ Representative images of $(D)$ quantified cells migrated through transwells towards serum, which was decreased in IL-6 knockout, TSC2-deficient MEFs compared to TSC2-deficient control MEFs. Scale bar $=100 \mathrm{um}$. $(E)$ OCR is decreased in TSC2deficient MEFs with IL-6 knockout compared to control cells. Data show measurements from the Seahorse extracellular flux analyzer using the MitoStress assay. $(F)$ Summary and statistical analysis of OCR results. (G) Basal ECAR is decreased in TSC2-deficient MEFs with IL-6 knockout MEFs compared to control. $\mathrm{mpH}$, milli-pH. $(H)$ Energy map showing the global bioenergetic status of TSC2-deficient MEFs with IL-6 knockout compared to control. Data presented as the mean \pm SD of three-four independent experiments. One-Way ANOVA and Student's $t$ test were used for statistical analysis. ${ }^{*} p<0.05,{ }^{* *} p<0.01,{ }^{* * *} p<0.001,{ }^{* * * *} p<$ 0.0001 . 
A

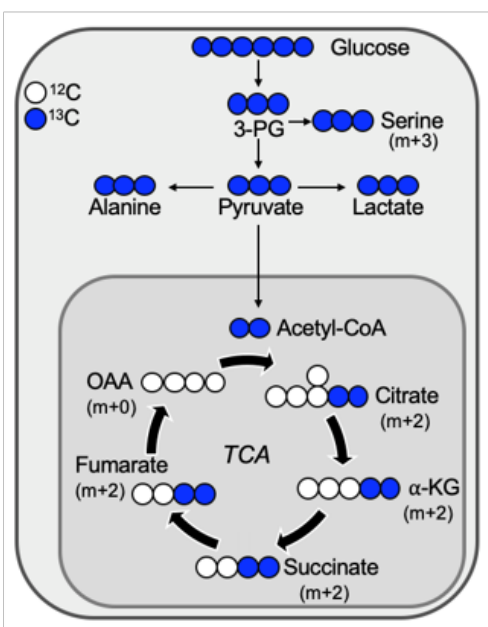

D

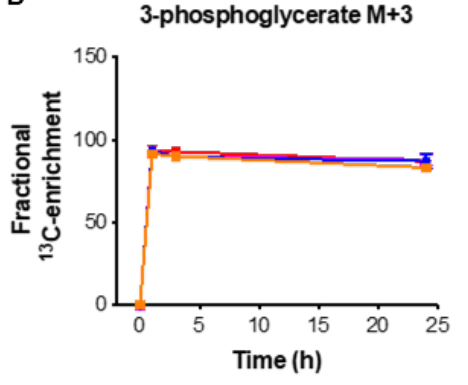

3-phosphoglycerate $M+3$

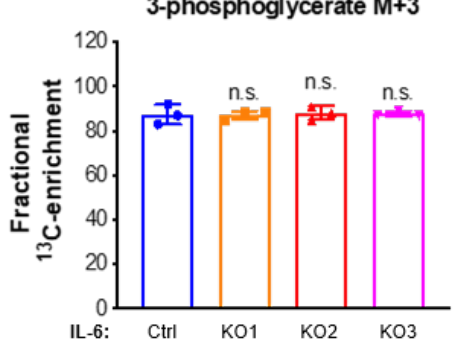

B
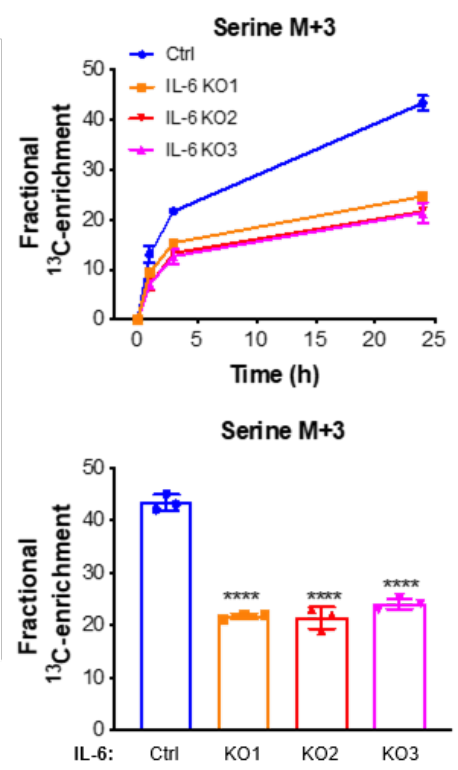

$E$

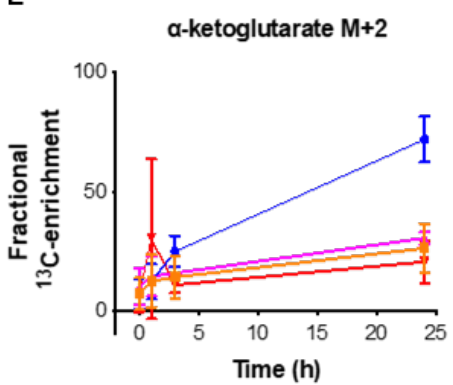

a-ketoglutarate $\mathrm{M}+2$

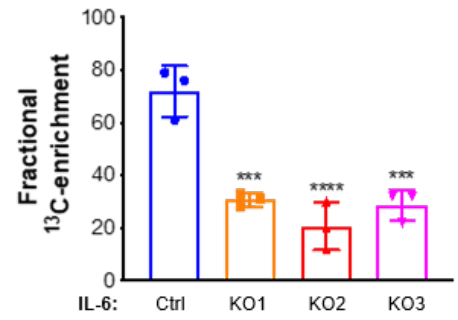

C
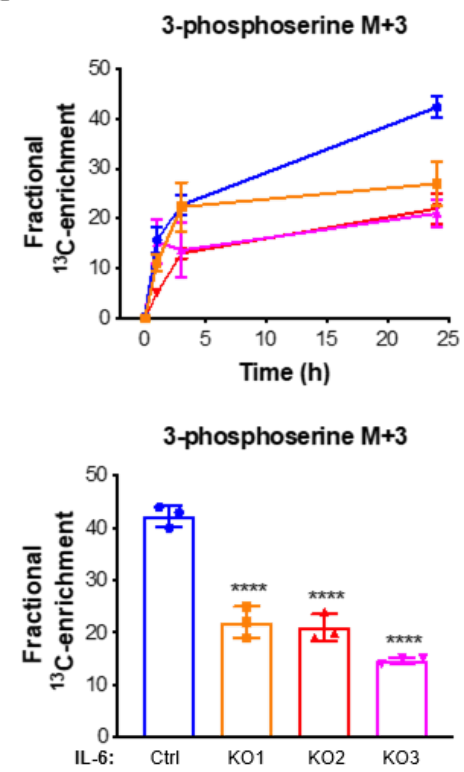

$\mathbf{F}$

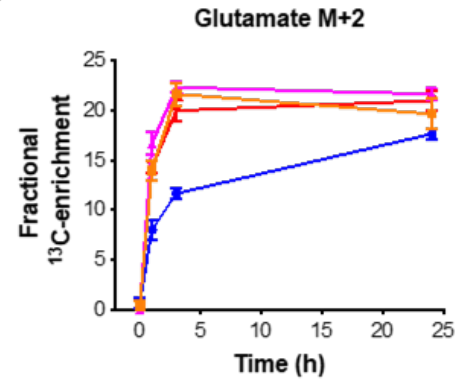

Glutamate $M+2$

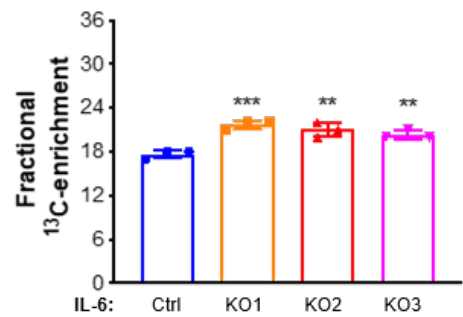

Figure 3. IL-6 regulates de novo serine synthesis in TSC2-deficient cells. $(A)$ Schematic of $\mathrm{U}-{ }^{13} \mathrm{C}$-glucose metabolism. The incorporation of ${ }^{13} \mathrm{C}$ atoms from ${ }^{13} \mathrm{C}_{6}$-glucose into citrate, $\alpha-$ ketoglutarate $(\alpha-K G)$, succinate, fumarate, and oxaloacetate $(O A A)$ are denoted as $M+n$, where $n$ is the number of ${ }^{13} \mathrm{C}$ atoms. $(B-F)$. Fractional enrichment of the $M+3$ isotopologues of serine, 3phosphoserine, and 3-phosphoglycerate or $\mathrm{M}+2$ isotopologues of a-ketoglutarate and glutamate in TSC2-deficient MEFs with IL-6 knockout compared to TSC2-deficient controls $(0,1,3,24$ hours after labelling; upper panels). Bar graphs show statistical analysis at the $24 \mathrm{~h}$ timepoint (bottom panels). Data presented as mean $\pm S D$ of three biological replicates. Statistical analysis performed by One-Way ANOVA, ${ }^{*} p<0.05,{ }^{* *} p<0.01,{ }^{* * *} p<0.001,{ }^{* * * *} p<0.0001$ as compared to control. 




$\mathrm{E}$

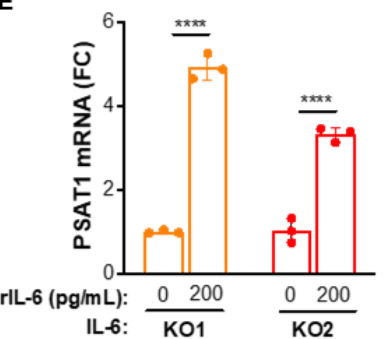

B

$\mathbf{F}$

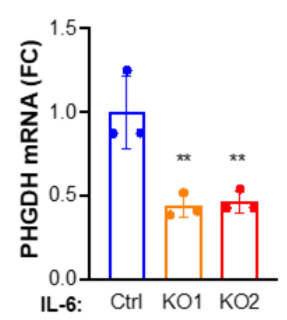

G
C D

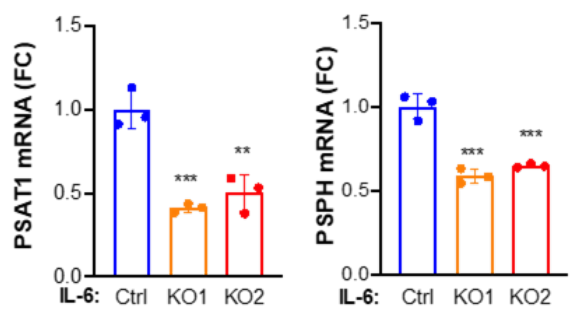

H



D
I

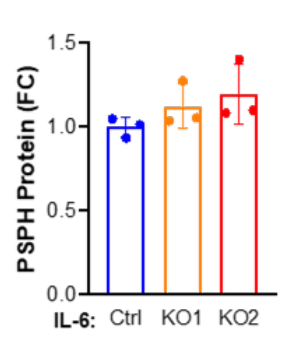

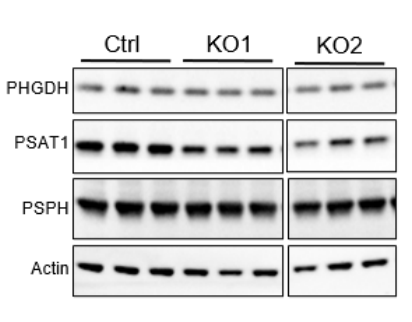

J
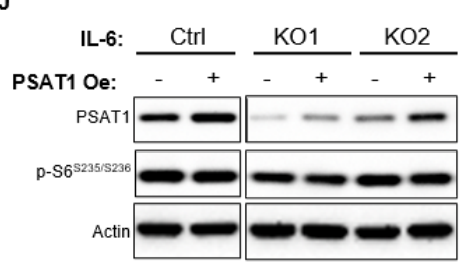

K

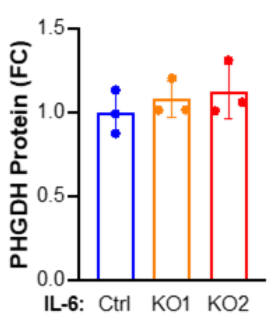

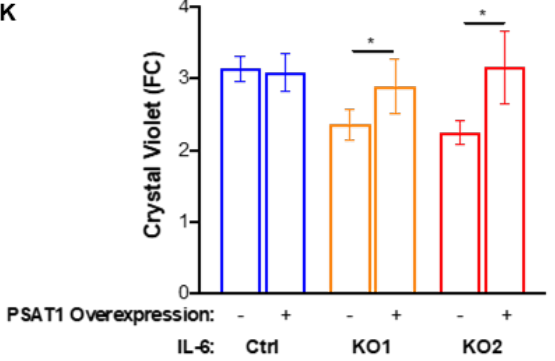

Figure 4. PSAT1 rescues proliferation of TSC2-deficient, IL-6 knockout cells. $(A)$ Diagram depicting interrelationships between glycolysis, the pentose phosphate pathway (PPP), de novo serine biosynthesis, and the tricarboxylic acid cycle (TCA). (B-D) PHGDH, PSAT1 and PSPH mRNA levels are decreased in TSC2-deficient, IL-6 knockout cells compared to TSC2-deficient control MEFs. (E) PSAT1 mRNA levels in IL-6 knockout, TSC2-deficient cells are rescued upon recombinant IL6 (rlL-6) treatment $(200 \mathrm{pg} / \mathrm{ml} ; 24 \mathrm{~h})$. (F-I) Western blot and densitometry showing expression of de novo serine biosynthesis enzymes and decreased PSAT1 expression in IL-6 knockout cells compared to control cells. Blots are from the same gel. Some lanes were cropped out for visualization purposes. $(J)$ Western blot confirming PSAT1 overexpression in TSC2deficient, IL-6 knockout cells and TSC2-deficient controls. Blots are from the same gel. $(K)$ PSAT1 overexpression rescues proliferation of IL-6 knockout, TSC2-deficient cells (72 h; fold change relative to day 0 , when the cells were washed and put into serum-free media). The data are presented as the mean \pm SD of three independent experiments. One-Way ANOVA was used for statistical analysis. ${ }^{*} p<0.05,{ }^{* *} p<0.01,{ }^{* * *} p<0.0001$ as compared to control. 
A

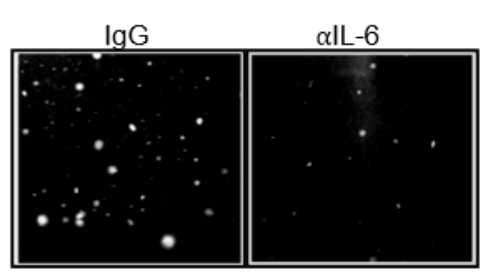

C

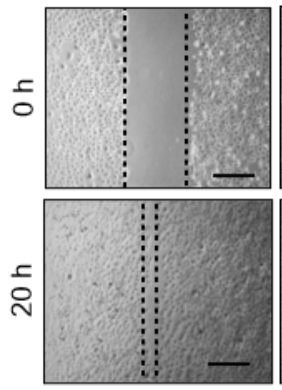

E

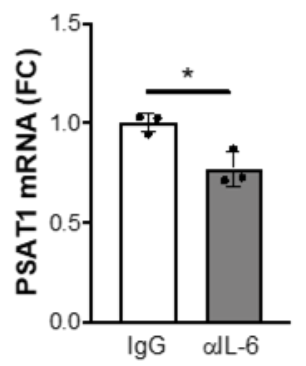

B
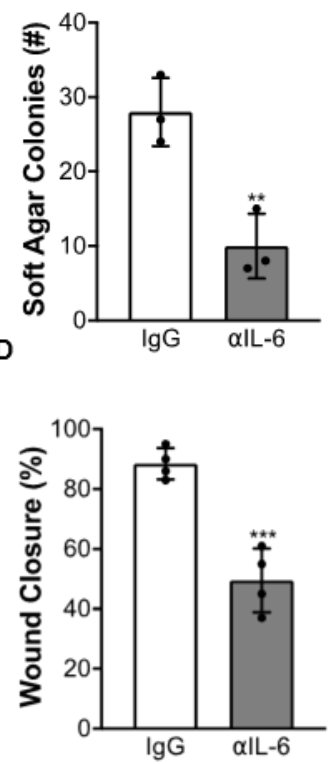

$\mathbf{F}$

Figure 5. alL-6 antibody suppresses proliferation, migration and PSAT1 expression in TSC2-deficient cells. $(A)$ Representative images and $(B)$ quantification of TSC2-deficient cell colonies grown for 14 days in soft agar and treated with alL-6 or IgG control antibody $(10 \mathrm{ug} / \mathrm{ml})$ in serum-free DMEM. Scale bar $=300 \mu \mathrm{m}$. $(C)$ Representative images and $(D)$ quantification of wound-healing assay performed on TSC2-deficient cells treated with alL-6 or IgG control antibody $(10 \mathrm{ug} / \mathrm{ml})$. alL-6 treatment decreases wound closure of TSC2-deficient cells compared to $\mathrm{lgG}$ control antibody after $20 \mathrm{~h}(\alpha \mathrm{lL}-6 ; 10 \mathrm{ug} / \mathrm{ml})$. Quantification of percent area filled between the two leading edges at $20 \mathrm{~h}$ compared to $0 \mathrm{~h}(0 \%$ filled). Scale bar $=150 \mu \mathrm{m}(E)$ PSAT1 mRNA and $(F)$ PSAT1 protein are decreased by alL-6 antibody $(10 \mathrm{ug} / \mathrm{ml} ; 48 \mathrm{~h})$ in TSC2-deficient cells. Cells were grown in serum-free DMEM for the duration of the experiments. The data are presented as mean \pm SD of three independent experiments. Student's $t$ test was used for statistical analysis with ${ }^{*} p<0.05,{ }^{* *} p<0.01,{ }^{* *} p<0.001$. 
A

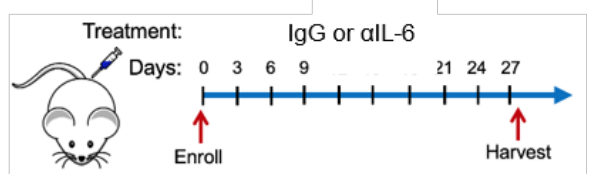

B
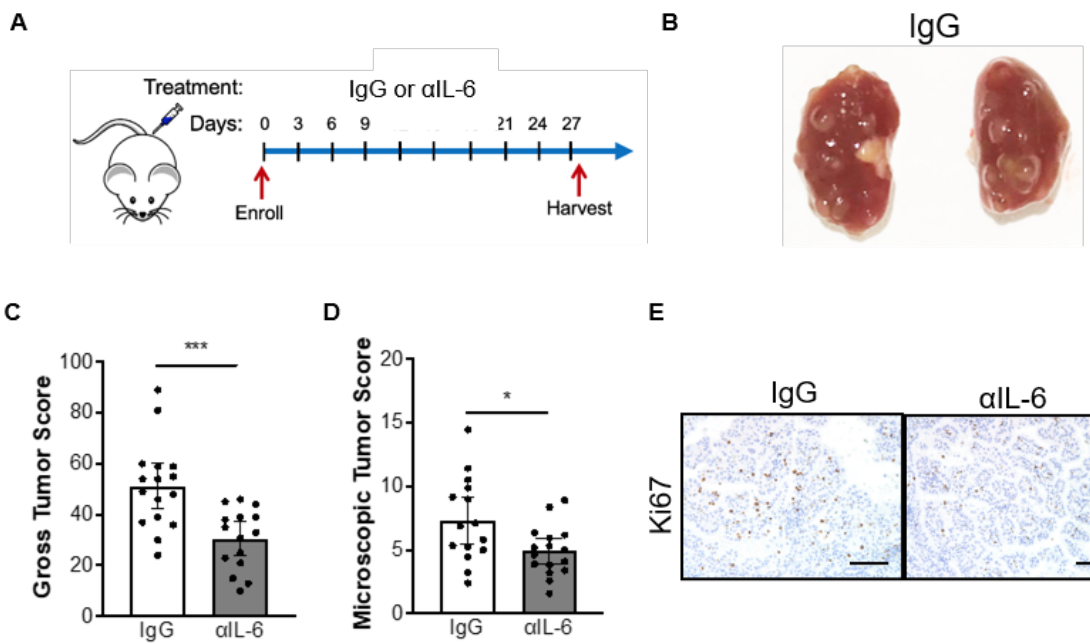

E

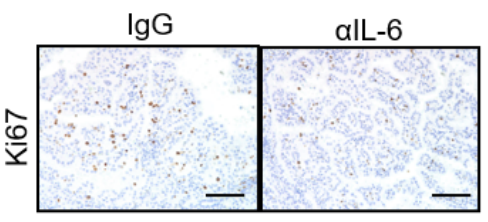

$\alpha$ IL-6

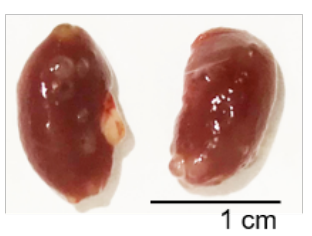

F

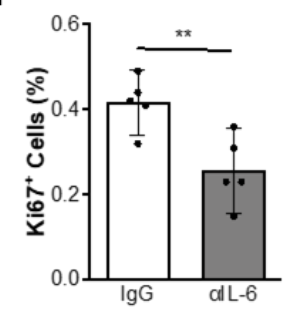

G

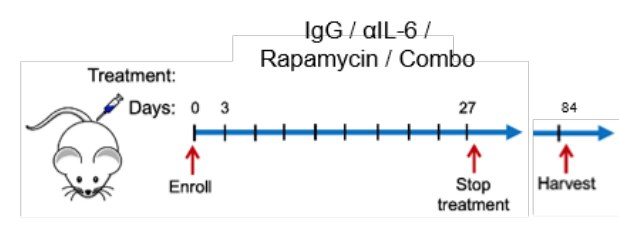

H

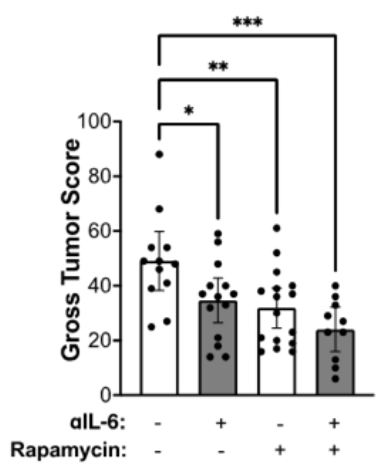

I

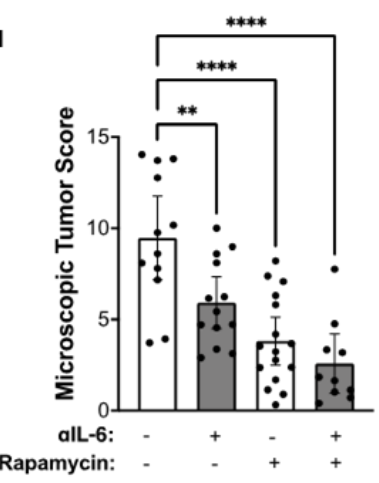

Figure 6. alL-6 antibody suppresses renal cystadenoma formation in $T s c 2^{+/-}$mice. $(A)$ Experimental design of alL- 6 antibody treatment. Tsc2+/- mice were injected intraperitoneally with IgG or alL-6 antibody (200 ug/mouse, three times per week) for one month and then harvested 24-48h after the last injection. (B) Representative kidneys of $T s c 2^{+/-}$mice injected intraperitoneally with IgG or alL-6 antibody. (C) alL-6 antibody decreased the gross tumor score and $(D)$ microscopic tumor score of $T s c 2^{+/-}$kidneys compared to IgG treated. (E) Representative images of Ki67 staining in Tsc2+/- renal tumors and $(F)$ quantification showing decreased proliferation in mice treated with alL-6 antibody compared to IgG control mice. (G) Experimental design to determine the duration of therapeutic benefit following alL-6, rapamycin, or combination treatments. Tsc2+/- mice were treated for one month and then harvested 2 months after the final injection. $(H)$ Gross tumor score and $(I)$ microscopic tumor score of kidneys from $\mathrm{Tsc}^{+/-}$mice treated with IgG, alL-6 (200ug/mouse, three times/week), rapamycin (3mg/kg three times/week) or combination, two months after treatment cessation. Data are presented as the mean $+/-95 \%$ $\mathrm{Cl}$, each dot represents one kidney, Statistical analysis was performed with Student's t test or One-Way ANOVA. Scale bar $=100 \mu \mathrm{m}$. 
A

\begin{tabular}{|c|c|c|c|c|c|c|c|c|c|c|c|}
\hline & A & B & c & D & $\mathrm{E}$ & $\mathbf{F}$ & G & $\mathrm{H}$ & 1 & $\mathrm{~J}$ & K \\
\hline 1 & POS & POS & POS & POS & NEG & NEG & $\begin{array}{l}\text { ENA-78 } \\
\text { (CXCL5) }\end{array}$ & G-CSF & GM-CSF & GRO a/b/g & \begin{tabular}{|l} 
GRO alpha \\
(CXCL1)
\end{tabular} \\
\hline 2 & $\begin{array}{l}\text { 1-309 } \\
\text { (CCL1) }\end{array}$ & $\begin{array}{l}\text { IL-1 alpha } \\
\text { (lL-1 F1) }\end{array}$ & $\begin{array}{l}\text { IL-1 beta } \\
\text { (L-1 F2) }\end{array}$ & IL-2 & IL-3 & IL-4 & IL-5 & IL-6 & IL-7 & $\begin{array}{c}\text { IL-8 } \\
\text { (CXCL8) }\end{array}$ & IL-10 \\
\hline 3 & $\begin{array}{c}\text { IL-12 } \\
\text { p40/p70 }\end{array}$ & IL-13 & IL-15 & IFN-gamma & $\begin{array}{l}\text { MCP-1 } \\
\text { (CCL2) }\end{array}$ & $\begin{array}{l}\text { MCP-2 } \\
\text { (CCL8) }\end{array}$ & $\begin{array}{l}\text { MCP3 } \\
\text { (CCL7) }\end{array}$ & M-CSF & $\begin{array}{c}\text { MDC } \\
\text { (CCL22) }\end{array}$ & $\begin{array}{c}\text { MIG } \\
\text { (CXCL9) }\end{array}$ & $\begin{array}{l}\text { MIP-1 beta } \\
\text { (CCL4) }\end{array}$ \\
\hline 4 & $\begin{array}{l}\text { MIP-1 delta } \\
\text { (CCLL15) }\end{array}$ & $\begin{array}{l}\text { RANTES } \\
\text { (CCL5) }\end{array}$ & SCF & SDF-1 alpha & $\begin{array}{l}\text { TARC } \\
\text { (CCL17) }\end{array}$ & TGF beta 1 & TNF alpha & \begin{tabular}{|l} 
TNF beta \\
(TNSF1B)
\end{tabular} & EGF & IGF-1 & Angiogenin \\
\hline 5 & OSM & TPO & VEGF-A & PDGF-BB & Leptin & BDNF & $\begin{array}{c}\text { BLC } \\
\text { (CXCL13) }\end{array}$ & $\begin{array}{l}\text { Ck beta 8-1 } \\
\text { (CCL23) }\end{array}$ & $\begin{array}{l}\text { Eotaxin-1 } \\
\text { (CCL11) }\end{array}$ & $\begin{array}{l}\text { Eotaxin-2 } \\
\text { (CCL24) }\end{array}$ & $\begin{array}{l}\text { Eotaxin-3 } \\
\text { (CCL26) }\end{array}$ \\
\hline 6 & FGF-4 & FGF-6 & $\begin{array}{l}\begin{array}{l}\text { FGF-7 } \\
\text { (KGF) }\end{array} \\
\end{array}$ & FGF-9 & $\begin{array}{l}\text { FLT-3 } \\
\text { Ligand }\end{array}$ & $\begin{array}{l}\text { Fractalkine } \\
\text { (CX3CL1) }\end{array}$ & $\begin{array}{c}\text { GCP-2 } \\
\text { (CXCL6) }\end{array}$ & GDNF & HGF & IGFBP-1 & IGFBP-2 \\
\hline 7 & IGFBP-3 & IGFBP-4 & IL-16 & $\begin{array}{c}\text { IP-10 } \\
\text { (CXCL10) }\end{array}$ & LIF & $\begin{array}{c}\text { LIGHT } \\
\text { (TNFSF14) }\end{array}$ & $\begin{array}{l}\text { MCP-4 } \\
\text { (CCL13) }\end{array}$ & MIF & $\begin{array}{c}\text { MIP-3 alpha } \\
\text { (CCL20) }\end{array}$ & $\begin{array}{l}\text { NAP-2 } \\
\text { (CXCL7) }\end{array}$ & NT-3 \\
\hline 8 & NT-4 & $\begin{array}{l}\text { OPN } \\
\text { (SPP1) }\end{array}$ & $\begin{array}{c}\text { OPG } \\
\text { (TNFRSF11B) }\end{array}$ & PARC & PLGF & TGF beta2 & TGF beta3 & TIMP-1 & TIMP-2 & POS & POS \\
\hline
\end{tabular}

B

$621-101$
Conditioned Media

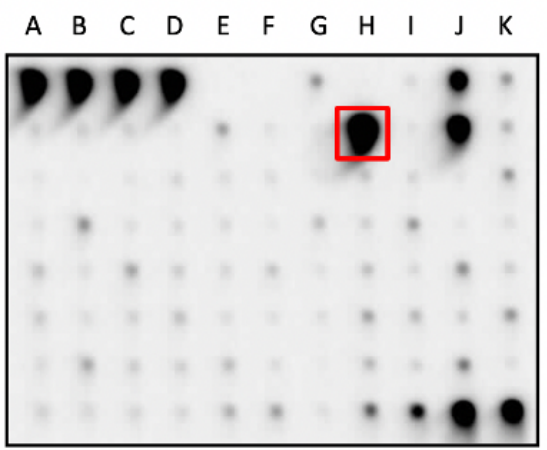

D

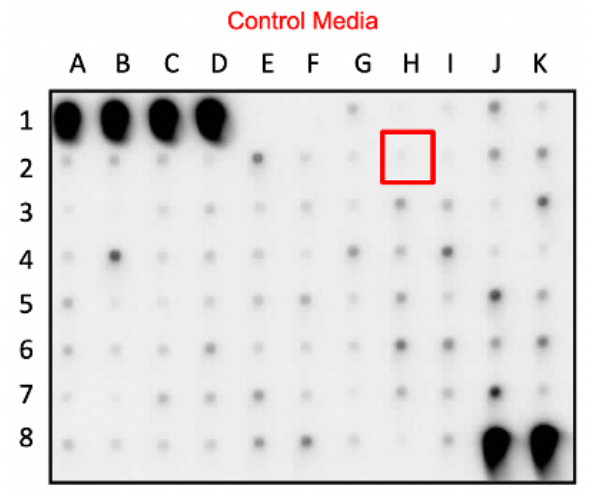

C

HEK-293

Conditioned Media

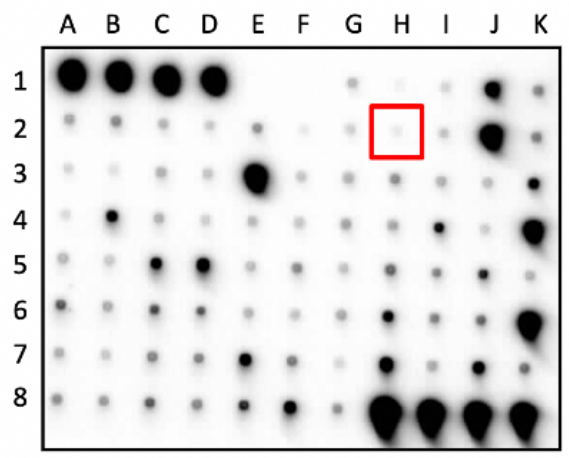

E

$\mathbf{F}$
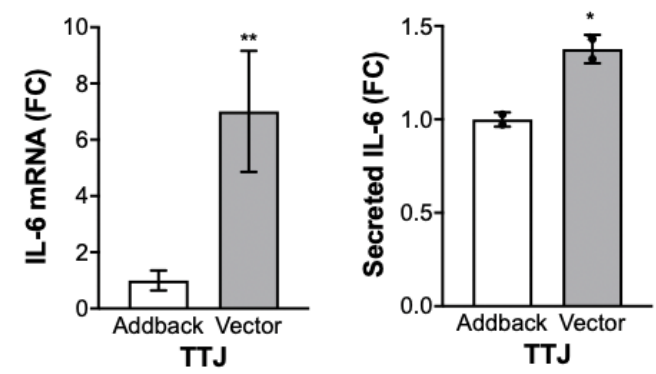

Fig. S1. Increased IL-6 secretion in conditioned media from TSC2-deficient cells. $(A)$ Layout of cytokine positions in RayBiotech Human Cytokine Array 5. (B) Cytokine array showing IL-6 expression is elevated in conditioned media from TSC2-deficient, patient-derived angiomyolipoma cells (621-101). (C) Cytokine array showing IL-6 expression in conditioned media from human embryonic kidney HEK293 cells. (D) Media incubated without cells was used as a blank control for the cytokine profile. $(E) I L-6$ mRNA expression is increased in TSC2deficient TTJ cells compared to TSC2 addback cells. $(F)$ Secreted IL-6 is increased in conditioned media from TSC2-deficient TTJ cells compared to TSC2 addback cells. 

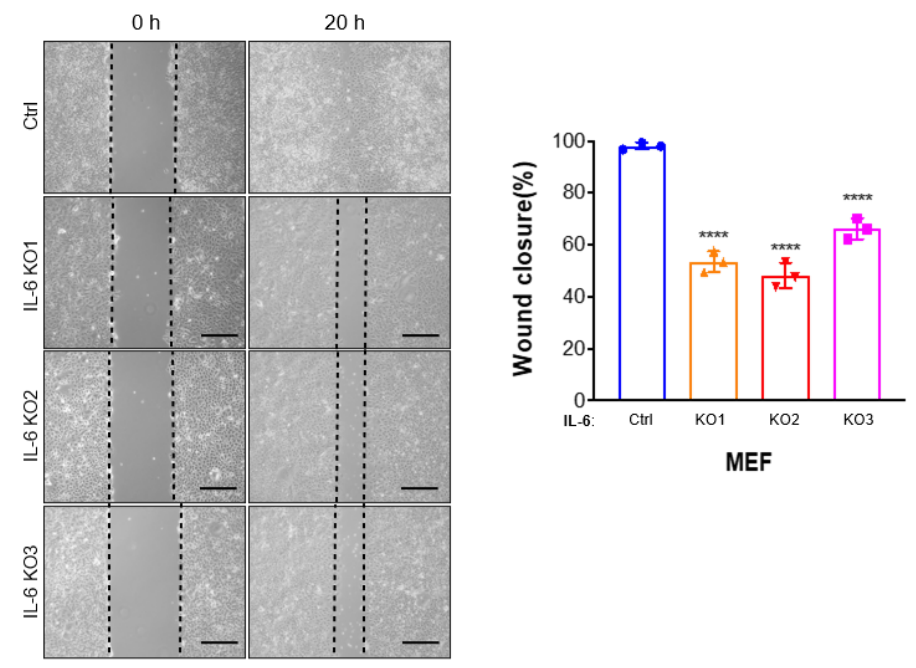

c
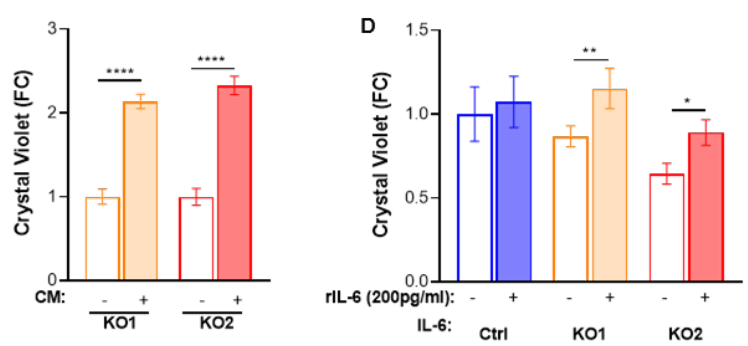

E
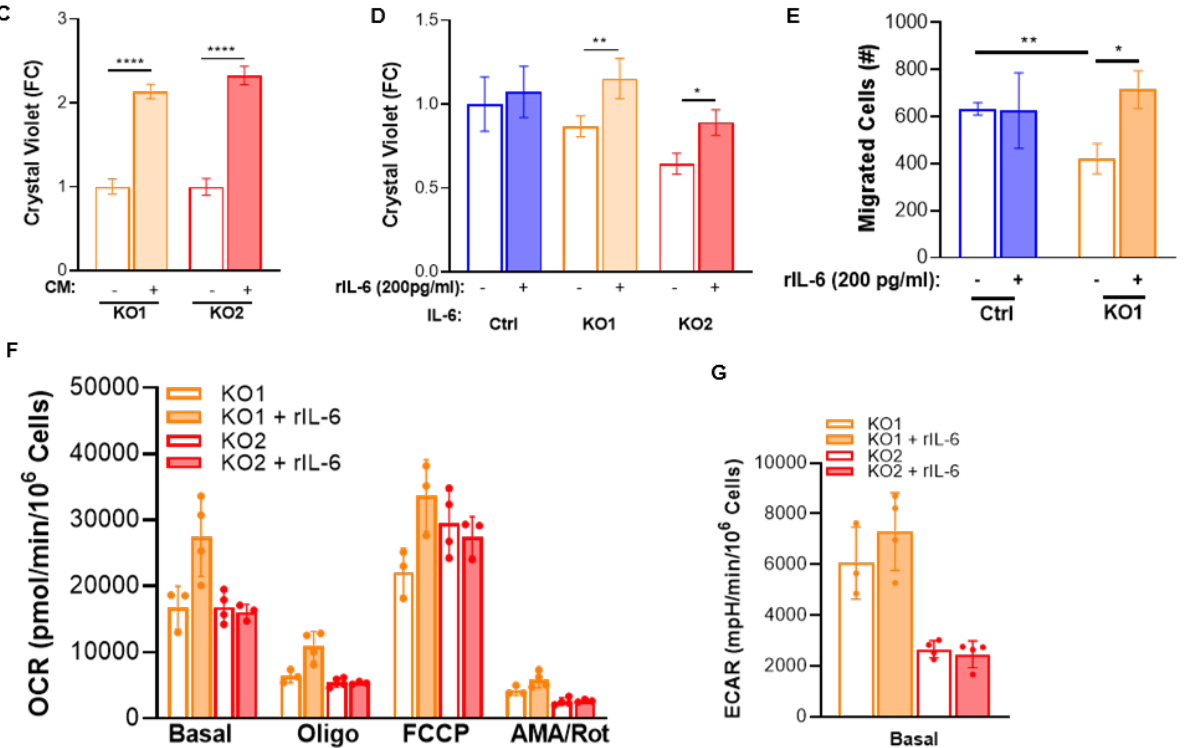

G

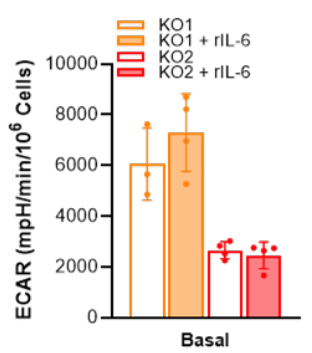

Fig. S2. Rescue of IL- 6 knockout cell proliferation, migration and bioenergetic assays. $(A)$ IL-6 knockout using three separate CRISPR/Cas9 clones shows decreased wound closure compared to control cells. $(B)$ Quantification of percent area filled between the two leading edges at $20 \mathrm{~h}$ compared to $0 \mathrm{~h}$ ( $0 \%$ filled). (C) The proliferation at $96 \mathrm{~h}$ of IL-6 knockout, TSC2 deficient cells was rescued by conditioned media, generated by concentrating serum free media from TSC2 deficient cells at 24 hours. (D) The proliferation at 72h of IL-6 knockout, TSC2 deficient cells was rescued by rlL-6 $(200 \mathrm{pg} / \mathrm{ml})$ treatment in serum free conditions. (E) rlL-6 rescued transwell migration of IL-6 knockout cells. Cells were pre-treated with rlL-6 (24h, 200pg/ml) and then seeded into the apical side of the transwell with rlL-6 or not treated controls. F) OCR and $(E)$ ECAR are unchanged in IL6 KO cells upon treatment with recombinant IL6. Data show measurements from the Seahorse extracellular flux analyzer using the MitoStress assay. The data are presented as mean $\pm S D$ of three independent experiments. Students $t$ test or One-Way ANOVA were used for statistical analysis. ${ }^{*} p<0.05,{ }^{* *} p<0.01,{ }^{* * * *} p<0.0001$. 
A

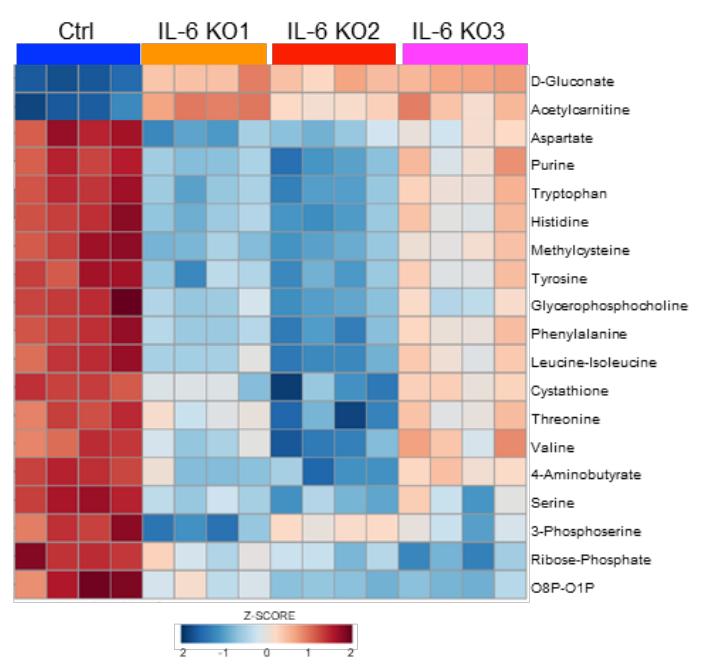

C
B

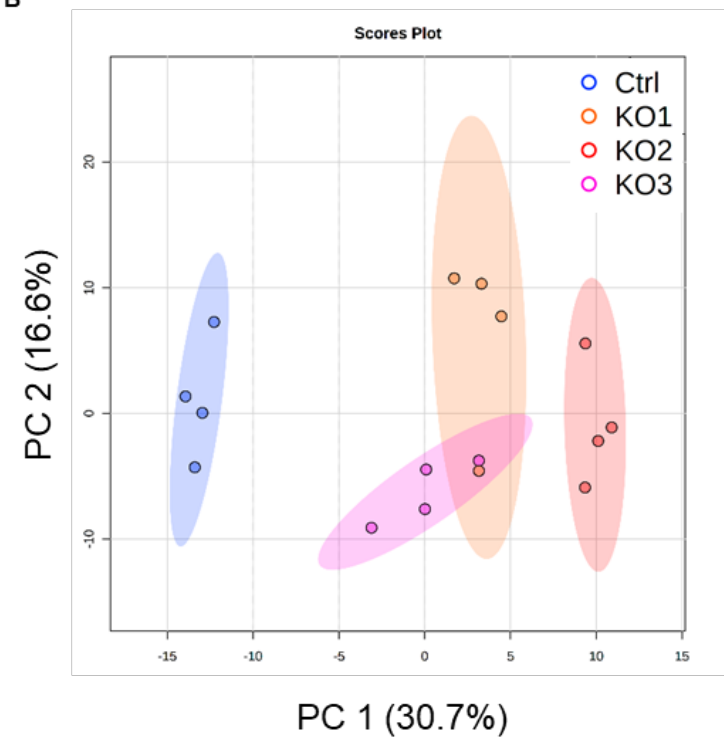

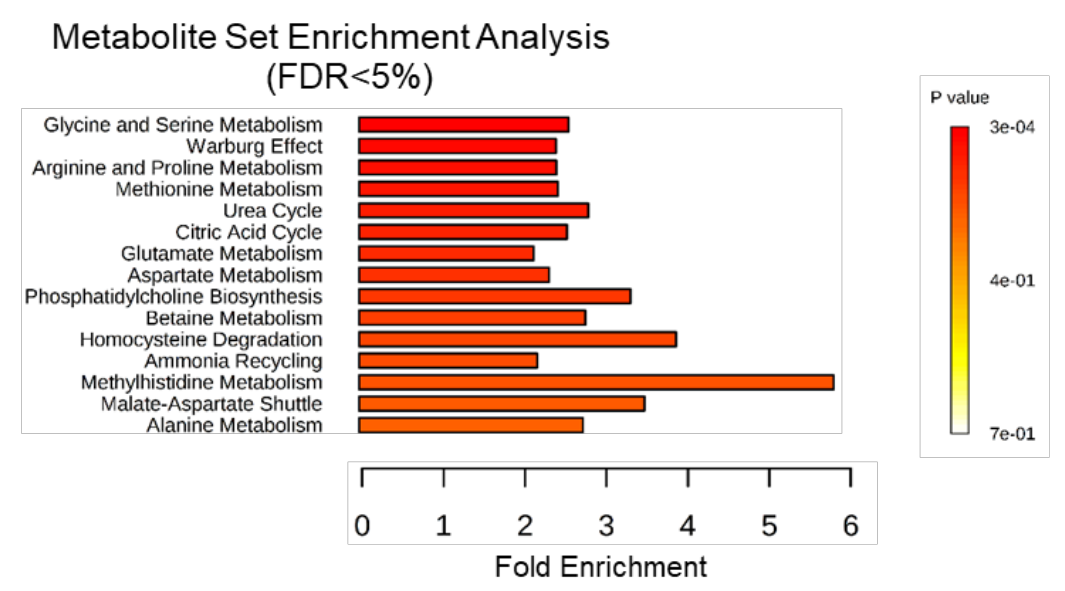

Fig. S3. IL-6 knockout shows a distinct metabolic signature. $(A)$ Hierarchical clustering and heatmap showing the top 20 differential metabolites in three IL-6 knockout TSC2-deficient MEF clones compared to control. $(B)$ Principal component analysis shows that the metabolism of the three IL-6 knockout clones is distinctive from control cells. (C) Metabolite Set Enrichment Analysis (MSEA) of differentially regulated metabolic pathways upon IL-6 knockout with false discovery rate (FDR) $<5 \%$ identifies Glycine and Serine Metabolism as the most significantly regulated pathway. Heatmaps and MSEA were generated using MetaboAnalyst. 



Fig. S4. IL-6 knockout decreases serine in TSC2-deficient cells. (A-M) Metabolites of de novo serine biosynthesis, pentose phosphate pathway and TCA cycle quantified by LC/MS were differentially regulated in IL-6 knockout, TSC2-deficient MEFs compared to controls. Data presented as individual values with box and whisker plot showing mean \pm minimum and maximum value of four biological replicates. Statistical analysis was performed by One-Way ANOVA ${ }^{*} p<0.05,{ }^{* *} p<0.01,{ }^{* * *} p<0.001,{ }^{* * * *} p<0.0001$ as compared to control. 
A



C

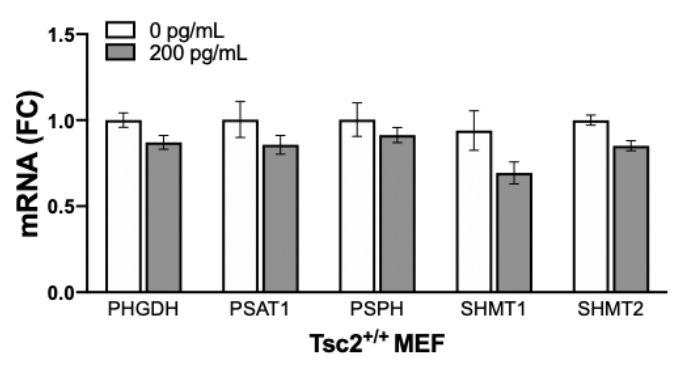

E

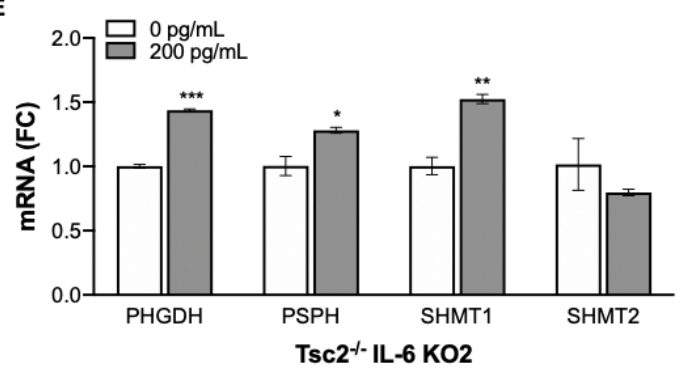

B

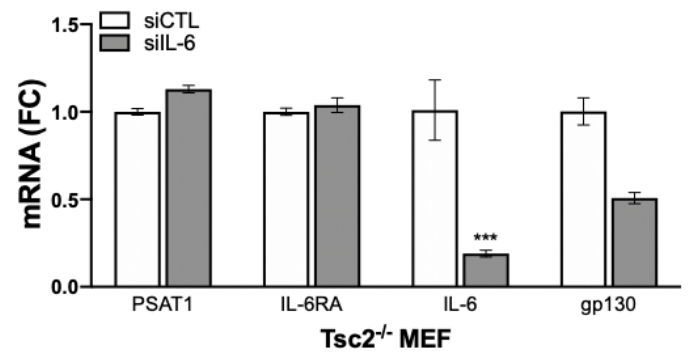

D

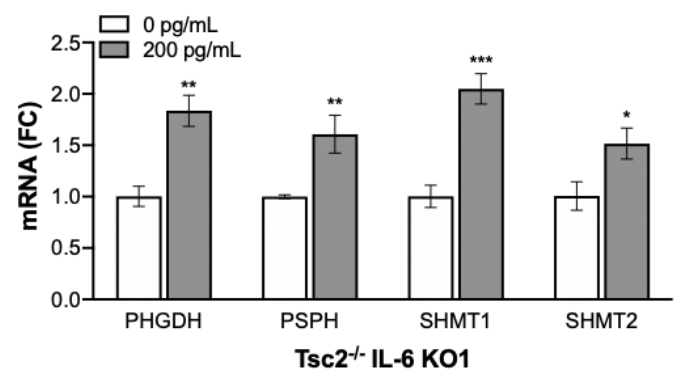

Fig. S5. IL-6 modulates serine biosynthesis genes in a TSC2-dependent manner. (A) IL-6 receptor knockdown using siRNA decreased PSAT1 mRNA expression in TSC2-deficient cells. (B) PSAT1 mRNA expression is unchanged by IL-6 knockdown using siRNA in TSC2-deficient cells. (C) Recombinant IL-6 treatment has no impact on de novo serine biosynthesis genes in Tsc2 wildtype cells (IL-6; 200pg/ml; 24 hours). ( $D$ and $E$ ) Recombinant IL-6 treatment of IL-6 knockout, Tsc2-deficient cells increased the mRNA expression of serine biosynthesis genes (IL-6; $200 \mathrm{pg} / \mathrm{ml} ; 24$ hours). The data are presented as mean \pm SD of three independent experiments. Student's t test was used for statistical analysis with ${ }^{*} p<0.05,{ }^{* *} p<0.01,{ }^{* * *} p<0.001$. 
A

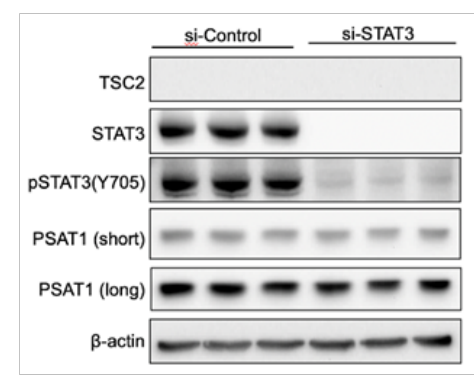

B

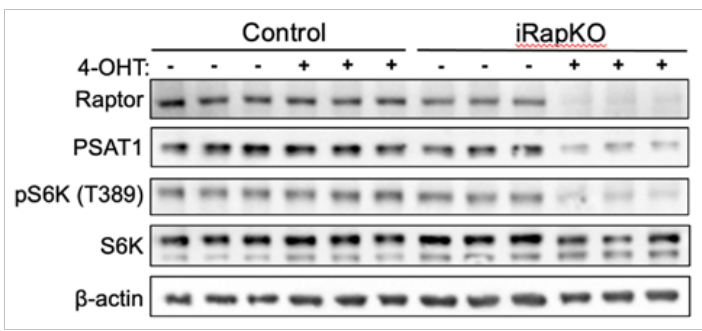

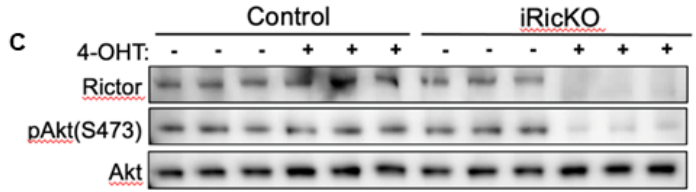

PSAT1 (short) $m=m=m-m=m-m$

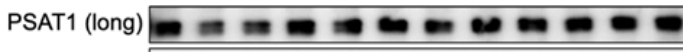

$\beta$-actin $-\cdots-\cdots-\cdots$

$\mathbf{F}$

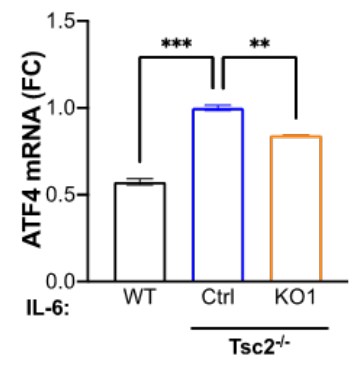

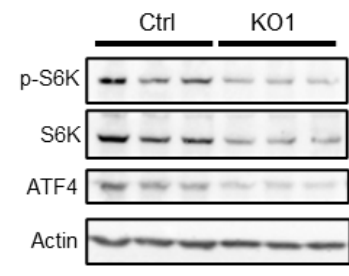

D

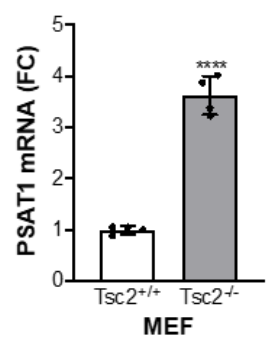

H

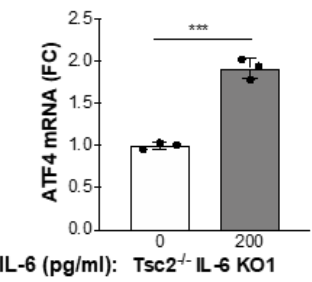

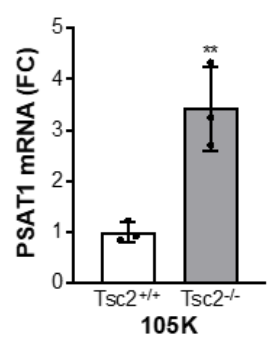

I

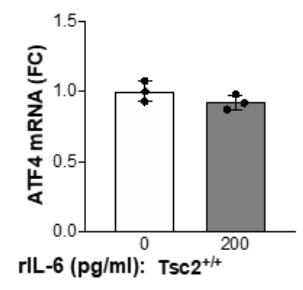

Fig. S6. PSAT1 is regulated in a STAT3-independent mTORC1-dependent manner. $(A)$ Western blotting showing that inhibition of STAT3 using siRNA did not impact PSAT1 protein expression (72h siRNA, serum-free last 24h). (B) mTORC1 inhibition using inducible Raptor knockout cells decreased PSAT1 protein levels. (C) mTORC2 inhibition using inducible Rictor knockout had no impact on PSAT1 protein levels. (D) PSAT1 mRNA levels are increased in TSC2-deficient MEFs compared to TSC2-expressing MEFs. (E) PSAT1 mRNA levels are increased in TSC2-deficient mouse kidney cystadenoma 105K cells compared to TSC2 reexpressing cells. $(F)$ ATF4 mRNA levels are increased in TSC2-deficient cells compared to TSC2-expressing cells, while IL-6 knockout decreased ATF4 expression. (G) Knockout of IL-6 inhibited phosphorylation of the mTORC1 target gene S6 kinase and the protein levels of ATF4. $(H)$ Recombinant IL-6 increased the mRNA expression of ATF4 in IL-6 knockout TSC2-deficient cells (rIL-6; $200 \mathrm{pg} / \mathrm{ml} ; 24$ hours). (I) Recombinant IL-6 did not impact the expression of ATF4 in TSC2-expressing cells rlL-6; 200pg/ml; $24 \mathrm{~h}$ ). Data are presented as mean \pm SD of three independent experiments. Student's t test and One-Way ANOVA were used for statistical analysis with ${ }^{* *} p<0.01,{ }^{* * *} p<0.001,{ }^{* * * *} p<0.0001$. 
A

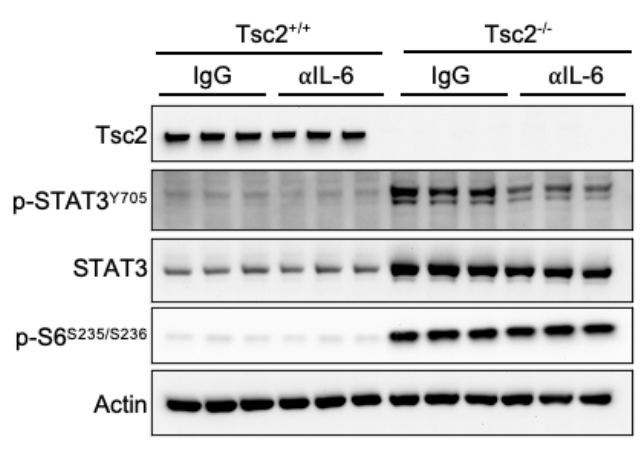

C

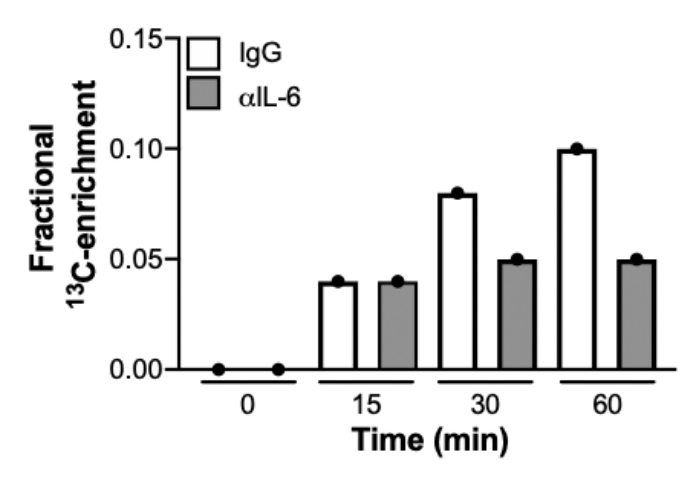

B

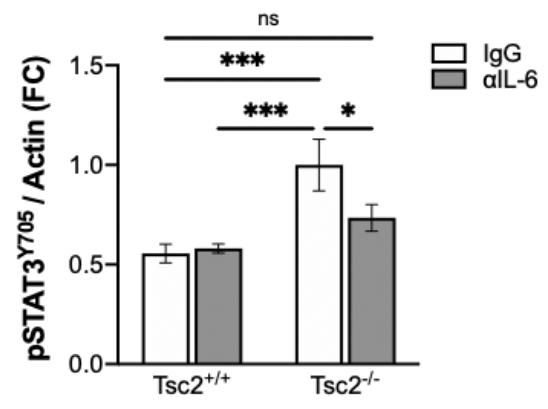

Fig. S7. alL-6 antibody reduced STAT3 signaling and serine synthesis in TSC2-deficient cells. $(A)$ Western blot and $(B)$ densitometry show that alL-6 antibody decreased phosphorylation of STAT3 ${ }^{Y 705}$ in TSC2-deficient cells (alL-6; 10ug/ml; $24 \mathrm{~h}$ ). (C) U- ${ }^{13} \mathrm{C}$ glucose tracing following alL-6 antibody decreased $M+3$ serine levels compared to $\mathrm{lg} G$ antibody control (10ug/ml). Data in $\mathrm{C}$ are derived from a single biological replicate for each treatment condition and time point. 
A PSAT1 Expression

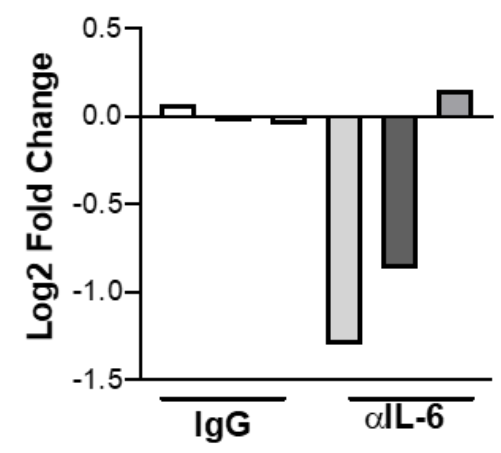

B


$\lg G$

C
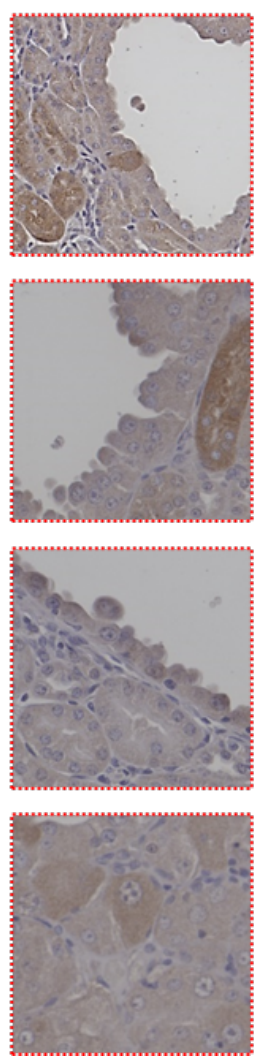

alL-6


Fig. S8. PSAT1 expression is reduced in renal lesions of $\mathrm{Tsc2}^{+/-}$mice treated with alL-6 antibody. (A) Semi-quantitative analysis of PSAT1 staining in three IgG and three alL-6 antibody treated mice (200 ug/mouse, three times/week). (B) Representative PSAT1 staining from IgG treated kidney with outlined area in red enlarged in image (Left column). Representative PSAT1 staining from alL-6 antibody kidney with outlined area in red enlarged in image (Right column). Crenal cyst, ca-cystadenoma, n-normal adjacent kidney, Scale bar $=100 \mu \mathrm{m}$. 